\title{
Fast-Forward Identification of Highly Effective Artificial Small RNAs Against Different Tomato spotted wilt virus Isolates
}

\author{
Alberto Carbonell,, ${ }^{1,+}$ Carmelo López, ${ }^{2}$ and José-Antonio Daròs ${ }^{1, \dagger}$ \\ ${ }^{1}$ Instituto de Biología Molecular y Celular de Plantas (Consejo Superior de Investigaciones Científicas-Universitat Politècnica de \\ València), 46022 Valencia, Spain; and ${ }^{2}$ Instituto de Conservación y Mejora de la Agrodiversidad Valenciana, Universitat \\ Politècnica de València, 46022 Valencia, Spain
}

Accepted 27 July 2018.

\begin{abstract}
Artificial small RNAs (sRNAs), including artificial microRNAs (amiRNAs) and synthetic trans-acting small interfering RNAs (syn-tasiRNAs), are used to silence viral RNAs and confer antiviral resistance in plants. Here, the combined use of recent high-throughput methods for generating artificial sRNA constructs and the Tomato spotted wilt virus (TSWV)-Nicotiana benthamiana pathosystem allowed for the simple and rapid identification of amiRNAs with high anti-TSWV activity. A comparative analysis between the most effective amiRNA construct and a syn-tasiRNA construct including the four most effective amiRNA sequences showed that both were highly effective against two different TSWV isolates. These results highlight the usefulness of this high-throughput methodology for the fast-forward identification of artificial sRNAs with high antiviral activity prior to time-consuming generation of stably transformed plants.
\end{abstract}

Artificial small RNAs (sRNAs) are 21-nucleotide (nt) RNAs designed to selectively silence transcripts, including viral RNAs, with high efficacy and specificity (Carbonell 2017a). In plants, artificial sRNAs include artificial microRNAs (amiRNAs) used to target single or sequence-related transcripts (Schwab et al. 2006) and synthetic trans-acting small

Corresponding authors: A. Carbonell; E-mail: acarbonell@ibmcp.upv.es and J.-A. Daròs; E-mail: jadaros@ibmcp.upv.es

Funding: This study was supported by Ministerio de Economía y Competitividad (Spain) grant BIO2014-54269-R (cofounded FEDER funds) and Ministerio de Ciencia, Innovación y Universidades (Spain) grant BIO201783184-R (cofounded FEDER funds) to J.-A. Daròs, and by Instituto Nacional de Investigaciones Agrarias grant RTA2013-00047-C02-02 (cofounded FEDER funds) grant RTA2013-00047-C02-02 to C. López. A. Carbonell was the recipient of an Individual Fellowship from the European Union Horizon 2020 research and innovation programme under the Marie Sklodowska Curie Individual Fellowship, European Union Horizon 2020 grant number 655841, and was selected in the Ramón y Cajal programme (RYC-2017-21648) from Ministerio de Ciencia, Innovación y Universidades (Spain).

*The $\boldsymbol{e}$-Xtra logo stands for "electronic extra" and indicates that four supplementary figures, four supplementary tables, three supplementary text files, and four supplementary datasets are published online.

(C) 2019 The American Phytopathological Society interfering RNAs (syn-tasiRNAs), which can target multiple sites within a transcript or multiple sequence-unrelated transcripts (de la Luz Gutiérrez-Nava et al. 2008; Montgomery et al. 2008a and b). Both classes of artificial sRNAs exploit endogenous sRNA-directed silencing pathways for their biogenesis and function and are synthesized in planta by expressing a transgene including a functional MIRNA or TAS precursor with modified miRNA or tasiRNA sequences, respectively. AmiRNAs arise from precursors with foldback structures processed by DICER-LIKE1 (DCL1), while syn-tasiRNAs are produced after processing of a TAS precursor by a miRNA/ARGONAUTE (AGO) complex, synthesis of double-stranded (ds)RNA from one of the cleavage products by RNA-DEPENDENT RNA POLYMERASE 6 (RDR6), and processing of such dsRNA by DLC4 into 21-nt phased syn-tasiRNAs in register with the miRNA-guided cleavage site. Importantly, despite differing in their biogenesis pathways, both classes of artificial sRNAs associate with an AGO protein, usually AGO1, to bind and silence highly sequence complementary transcripts (Carbonell 2017b). Methods to design, produce and validate artificial sRNA constructs have been recently optimized for highthroughput applicability and include i) a new generation of plant $B s a \mathrm{I} / c c d \mathrm{~B}(\mathrm{~B} / \mathrm{c})$ expression vectors for efficient, onestep cloning and high expression of artificial sRNAs (Carbonell et al. 2014, 2015) and ii) the P-SAMS tool for the automated design of highly specific plant artificial sRNAs (Fahlgren et al. 2016).

Tomato spotted wilt virus (TSWV) is the type species of the genus Tospovirus, which includes the only members of the family Bunyaviridae that infect plants (Plyusnin et al. 2012). TSWV, recently considered the second plant virus based on scientific and economic importance (Scholthof et al. 2011), has a wide host range that includes more than 1,000 species of weeds and ornamental and horticultural crops (Sherwood et al. 2003) and is transmitted by diverse species of thrips, such as Frankliniella occidentalis (Whitfield et al. 2005). The genome of TSWV consists of three negative or ambisense single-stranded RNAs, denoted segment large (L, $8.9 \mathrm{~kb})$, segment medium (M, $4.8 \mathrm{~kb})$, and segment small ( $\mathrm{S}, 2.9 \mathrm{~kb}$ ), based on their sizes (Kormlink 2011). Segment L is completely antisense and encodes the RNA-dependent RNA polymerase (RdRp); segment M is ambisense and encodes the putative movement protein NSm and the structural proteins $\mathrm{Gn} / \mathrm{Gc}$ involved in transmission by thrips; segment $\mathrm{S}$ is ambisense and encodes the nucleocapsid $\mathrm{N}$ protein and the silencing suppressor NSs (Kormlink 2011). 
Resistance to TSWV was obtained through the introgression of the two main resistance genes, $S w 5$ and $T s w$, in tomato and pepper, respectively. However, the constant emergence of resistance-breaking TSWV isolates has limited the durability of this type of resistance (Turina et al. 2016). Classic RNA interference (RNAi) approaches, such as overexpression of sense or hairpin transgenes including viral sequences (particularly from the $N$ and NSm genes) (Bucher et al. 2006; Jan et al. 2000; MacKenzie and Ellis 1992; Peng et al. 2014; Prins et al. 1996; Sonoda and Tsumuki 2004) and, more recently, amiRNAs targeting the $N$ gene (Mitter et al. 2016) have been also used to generate plant resistance to TSWV. Still, artificial sRNA-based strategies have not been systematically analyzed to confer highly-specific, potent and broad anti-TSWV resistance.

In this work, the goal was to explore, in a simple and timeeffective manner, whether amiRNAs and syn-tasiRNAs could be used to protect plants against diverse TSWV isolates. For that purpose, we combined the use of recently described highthroughput methods for the design and generation of highlyspecific artificial sRNA constructs (Carbonell et al. 2014, 2015, in press; Fahlgren et al. 2016) and the TSWV-Nicotiana benthamiana pathosystem to i) identify artificial sRNA sequences with high antiviral activity through a large functional screen of anti-TSWV amiRNAs, ii) analyze the antiviral activity of a syntasiRNA construct including the most effective artificial sRNA sequences identified in the preliminary amiRNA screen, and iii) functionally compare the anti-TSWV activity of amiRNAs and syn-tasiRNAs against two different TSWV isolates. The main steps followed in the present work are summarized in a workflow diagram in Figure 1 and include i) the design, selection, cloning, and functional analysis of anti-TSWV amiRNAs, ii) the generation and functional analysis of an anti-TSWV syntasiRNA construct including the most effective anti-TSWV amiRNA sequences, and iii) a comparative analysis of the activity of amiRNAs and syn-tasiRNAs against two different TSWV isolates.

\section{RESULTS}

\section{Rational design of amiRNAs against multiple TSWV isolates.}

To design amiRNAs against multiple TSWV isolates, we searched the National Center for Biotechnology Information (NCBI) database for all complete sequences corresponding to TSWV segments L, M, and S; 29, 65, and 61 sequences were collected, respectively. First, P-SAMS (Fahlgren et al. 2016) was used to design optimal amiRNAs against each TSWV segment with no off-targets in Solanum lycopersicum, the TSWV natural host. No results were obtained in P-SAMS designs, including either all 29, 65, or 61 sequences as input, suggesting that nucleotide variability within each segment was too high. Therefore, sequences corresponding to each segment from only six different variants isolated from S. lycopersicum in different regions of the world were used in subsequent P-SAMS designs (discussed below). A list of 143, 57, and 58 optimal amiRNAs specifically targeting segments L, M, and S, respectively, was obtained (Supplementary Dataset S1). Second, to identify those optimal amiRNAs targeting more conserved sites in the viral genome, the nucleotidic variability of each target site from all NCBI-collected sequences was estimated i) by first calculating the Shannon entropy value (Shannon 1997) of each TSWV genomic position (Fig. 2A) and ii) by next calculating the entropy value of each target site as the sum of the entropy values of all target site positions. Note that the entropy values of nucleotidic positions among the three TSWV segments is highly variable as shown in Figure 2A profiles, ranging from 0 to 1.97 (Supplementary Dataset S2). Five
amiRNAs targeting segment L, five amiRNAs targeting segment $\mathrm{M}$, and five amiRNAs targeting segment $\mathrm{S}$ were selected based on the following criteria (Table 1): i) amiRNAs extensively base-pair with target RNA (their P-SAMS score is 1 or close), ii) target sites have low variability across multiple isolates (their total entropy value is 0 or close, as shown in the entropy profiles of amiRNA target sites in Figure 2B), and iii) target sites are distributed along the corresponding segment in RNAs of both genomic and antigenomic polarities (Fig. 3). In particular, all amiRNAs against segment L target RdRp messenger RNA (mRNA), three and two amiRNAs against segment $M$ target NSm viral RNA (vRNA) and mRNA, respectively, and one and four amiRNAs against segment $S$ target $N$ mRNA and Nss vRNA, respectively. Importantly, none of the selected amiRNAs had significant off-targets in $N$. benthamiana, the experimental host chosen for the anti-TSWV amiRNA screening, based on TargetFinder (Fahlgren and Carrington 2010) computational prediction (Supplementary Table S2). Selected amiRNA sequences (Figs. 4A, 5A, and 6A; Table 1; Supplementary Text S3; Supplementary Dataset S3) were directly

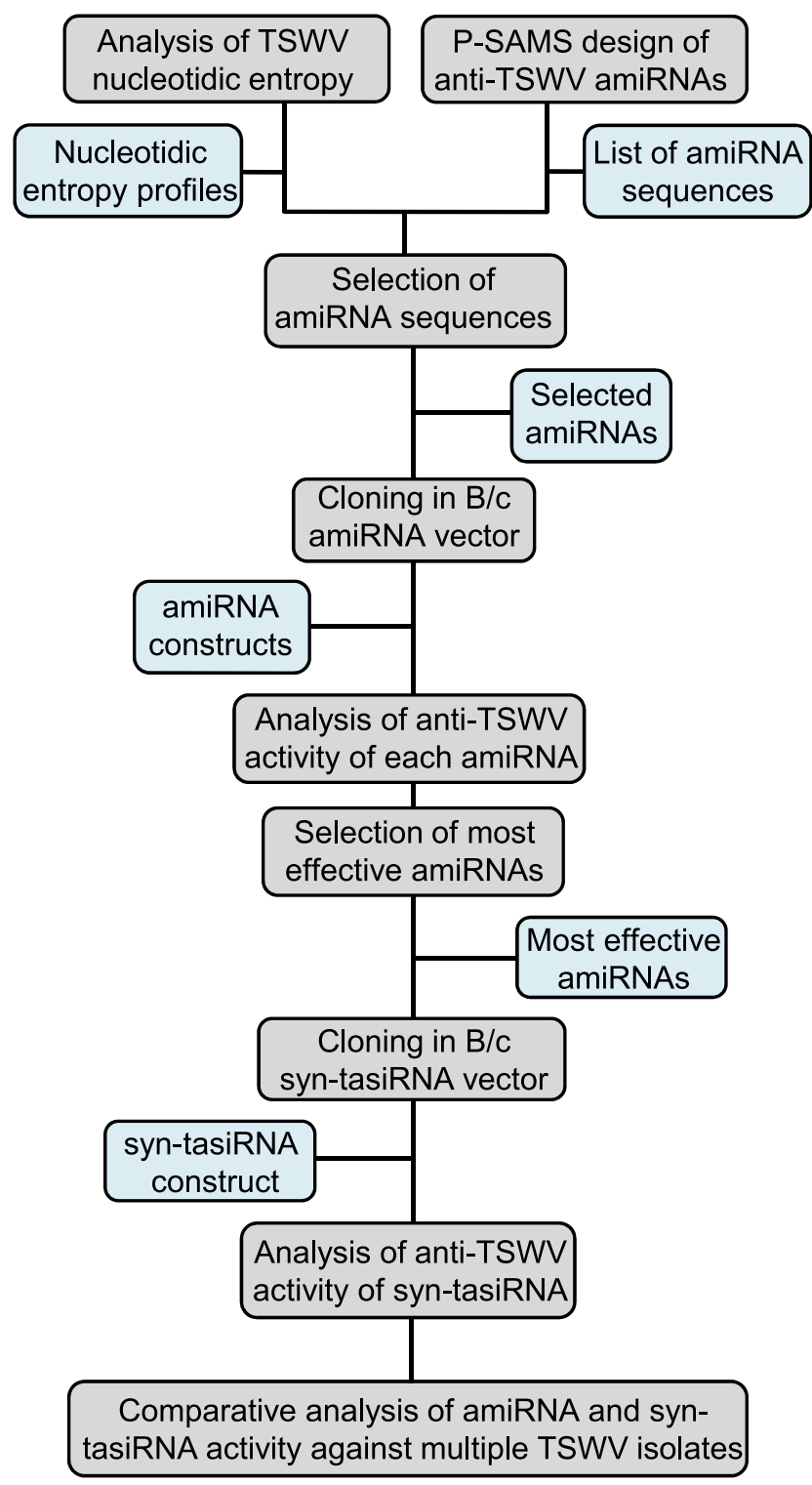

Fig. 1. Diagram of the steps for the design and synthesis of anti-Tomato spotted wilt virus (TSWV) artificial microRNAs (amiRNAs) and synthetic trans-acting small interfering RNAs (syn-tasiRNAs) and for the analysis of their antiviral activity. 
A

TSWV-L

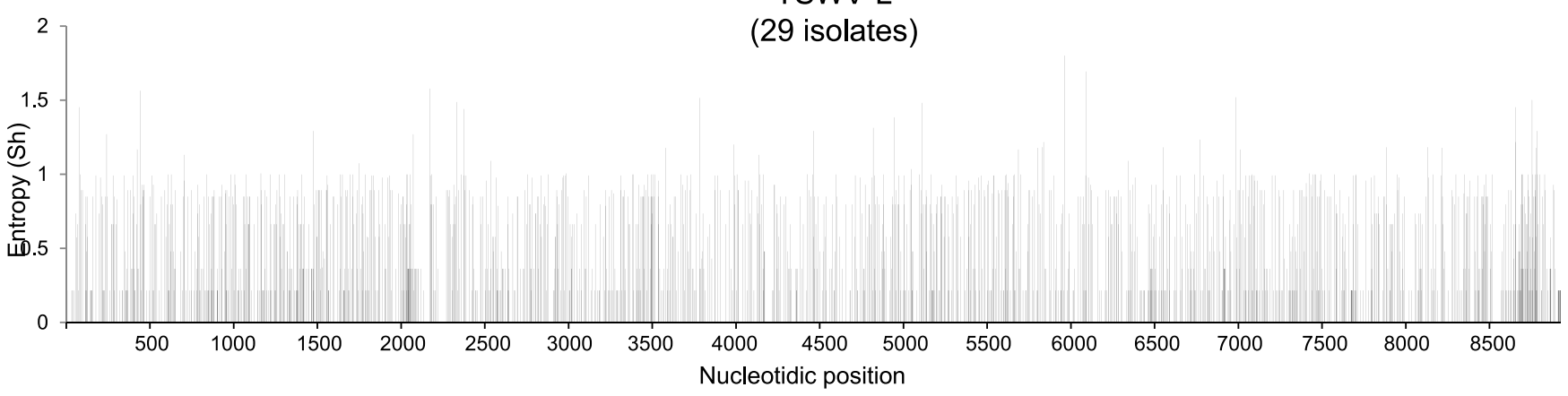

TSWV-M

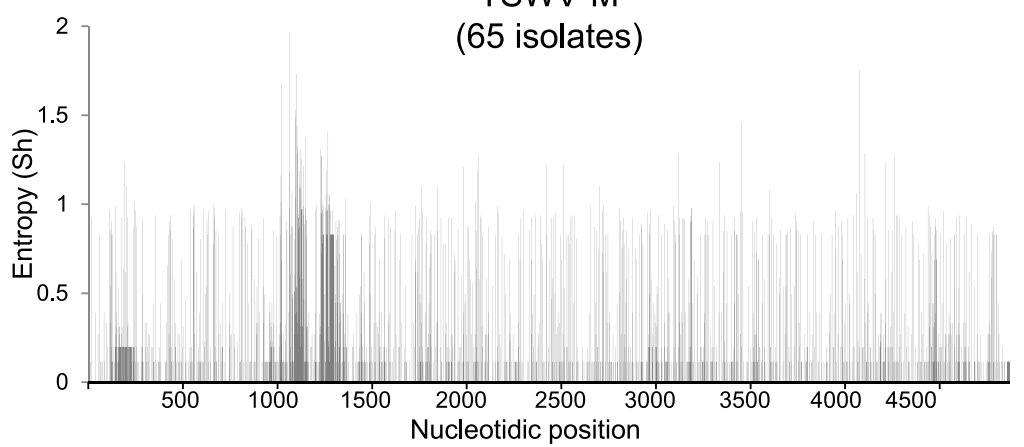

TSWV-S

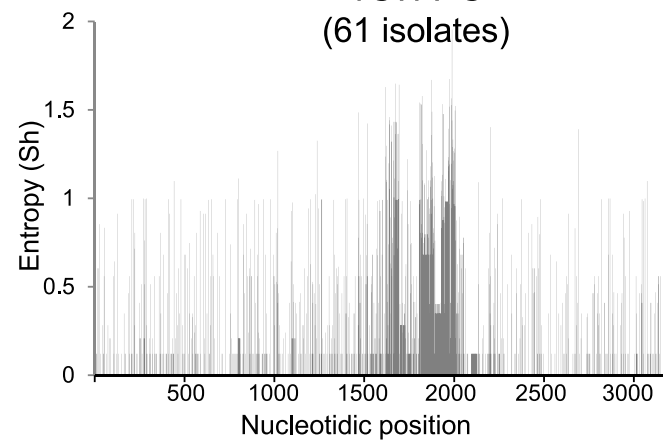

B
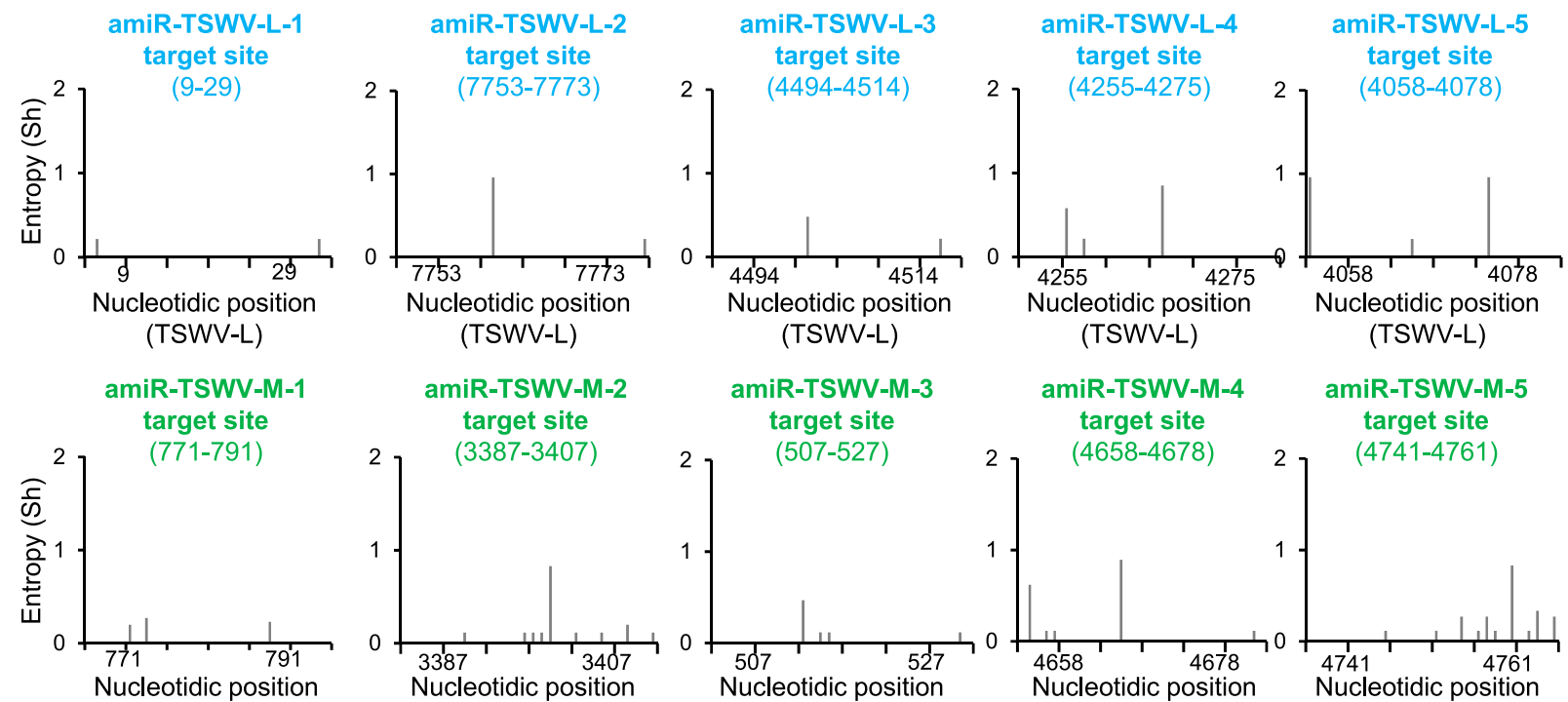

amiR-TSWV-M-2

target site

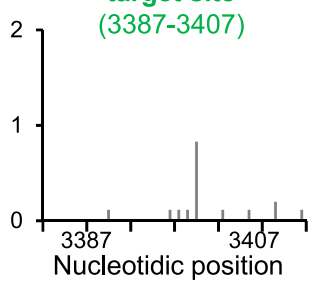

amiR-TSWV-M-3 target site

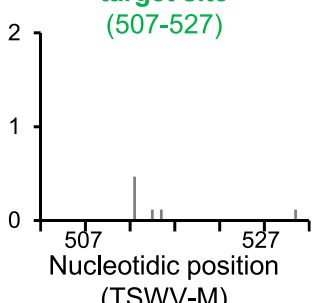

amiR-TSWV-M-4 target site

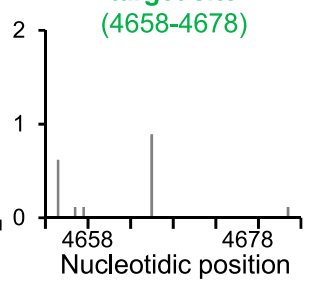

amiR-TSWV-M-5

eotidic positic
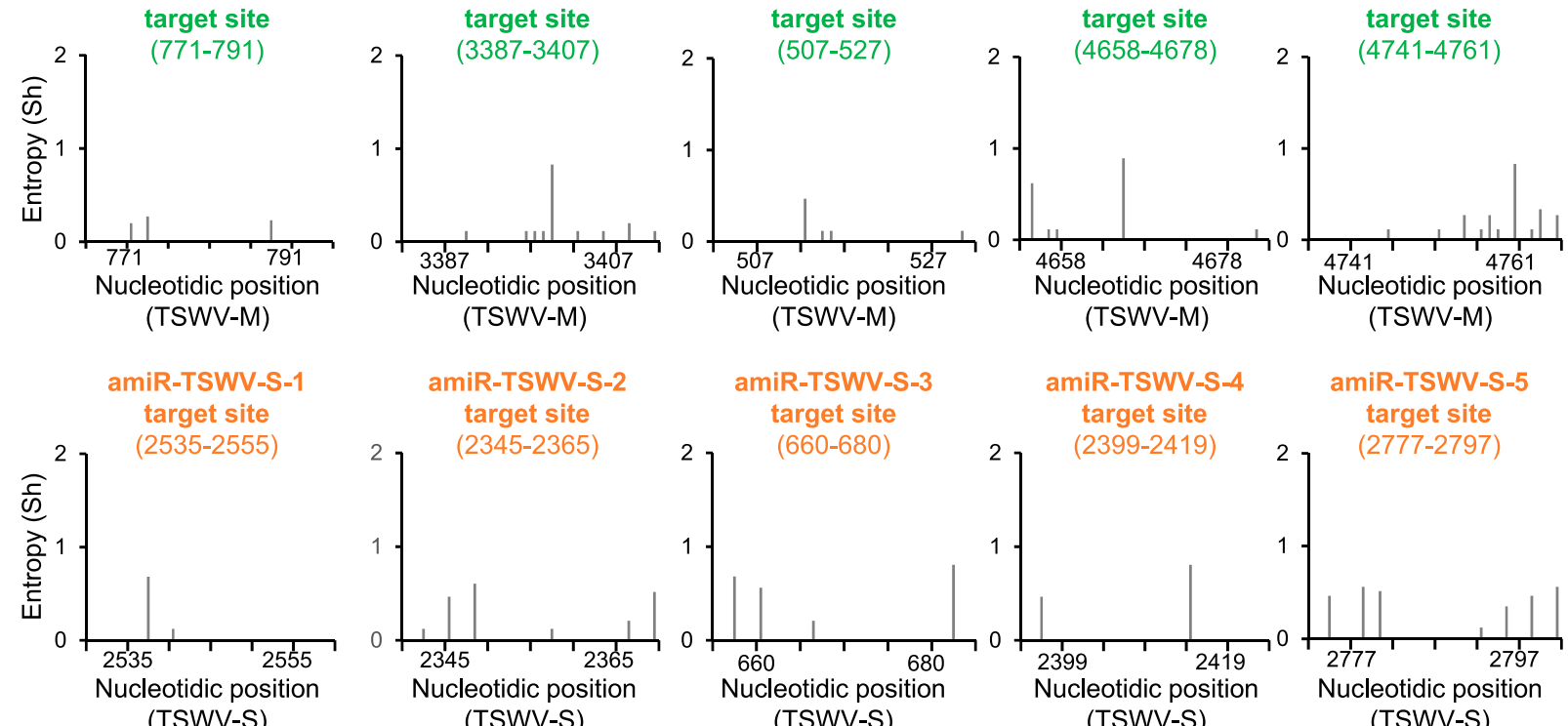

(TSWV-S)

(TSWV-S)

(TSWV-S)

(TSWV-S)

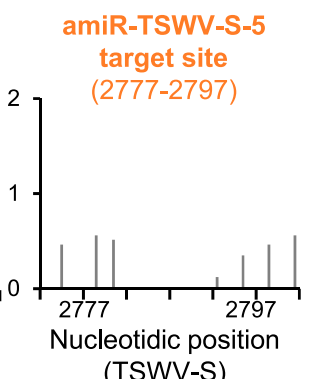

Fig. 2. Analysis of Tomato spotted wilt virus (TSWV) nucleotidic variability. A, Entropy profiles of TSWV segments L, M, and S. B, Entropy profiles of target sites from selected artificial microRNAs (amiRNAs). 
inserted into the $p M D C 32 B-A t M I R 390 a-B / c$ expression vector optimized for one-step cloning and high expression of amiRNAs in eudicots (Carbonell et al. 2014).

\section{Functional analysis of anti-TSWV amiRNAs.}

To begin, an extract from the well-characterized TSWV PVR isolate (Debreczeni et al. 2015) was previously titrated to determine the minimal amount of extract that elicited symptoms in all the plants mechanically inoculated. A dilution assay showed that only the undiluted extract, obtained at a 1:20 tissue/buffer ratio, induced symptoms in upper noninoculated tissues in $100 \%$ of the inoculated plants between 6 and 8 days postinoculation (dpi) (Supplementary Fig. S1). Therefore, the undiluted extract was used as TSWV PVR inoculum.

Next, the accumulation in planta of each amiRNA species was analyzed. Each amiRNA construct was independently agroinfiltrated in three different $N$. benthamiana plants, and infiltrated leaves were collected 2 days after agroinfiltration (dpa). Northern blot analysis of RNA preparations from agroinfiltrated leaves showed that amiRNAs accumulated to different levels (Supplementary Fig. S2). To functionally analyze selected amiRNAs, six $N$. benthamiana plants were agroinfiltrated independently with each of the anti-TSWV amiRNA constructs and were then mechanically inoculated 2 days later with the TSWV PVR extract. As negative controls, a set of plants was agroinfiltrated exclusively with the $35 S$ : GUS construct, and two other sets were agroinfiltrated independently with two anti-GUS control amiRNAs (Carbonell and Daròs 2017) and were subsequently inoculated with TSWV PVR. To determine the antiviral activity of amiRNAs, the appearance of typical TSWV-induced symptoms in inoculated tissues (local lesions) and in distant noninoculated tissues (leaf epinasty and chlorosis) was monitored. In inoculated tissues, the number of necrotic lesions was recorded. In upper noninoculated tissues, TSWV accumulation was analyzed by enzyme-linked immunosorbent assay (ELISA) at two timepoints (10 and $20 \mathrm{dpi}$ ).

Leaves agroinfiltrated with amiR-TSWV-L-3 and amiRTSWV-L-5 targeting the TSWV L segment showed very few or no lesions, respectively, when compared with leaves expressing the amiR-GUS control constructs. The rest of the anti-TSWV-L amiRNAs showed a similar number of necrotic lesions compared with controls (Fig. 4). Interestingly, at 10 dpi of all plants expressing amiR-TSWV-L-3 or amiR-TSWV-L-5, neither showed symptoms of infection (Fig. 4D and E) nor accumulated TSWV (Fig. 4F) in upper noninoculated leaves. In contrast, all plants expressing amiR-TSWV-L-1, amiR-TSWV-L-2, or amiR-TSWV-L-4 displayed symptoms, although several of them with a slight delay compared with controls (Fig. 4D and E), and accumulated high levels of TSWV (Fig. 4F). At the end of the experiment ( $20 \mathrm{dpi}$ ), all plants were positive by ELISA, except plants expressing amiR-TSWV-L-5 and half of the plants expressing amiR-TSWV-L-3, which did not show symptoms (Fig. 4E; Table 2). The other half of plants expressing amiRTSWV-L-3 showed symptoms at $13 \mathrm{dpi}$, almost a week later than control plants (Fig. 4E).

In the case of amiRNAs targeting the $M$ fragment (Fig. 5A), leaves expressing amiR-TSWV-M-3 and amiR-TSWV-M-1 showed very few or no lesions, respectively, while leaves expressing the rest of anti-TSWV-M amiRNAs displayed a similar number of lesions as amiR-GUS controls (Fig. 5B and C). Importantly, the analysis of upper noninoculated tissue at 10 dpi revealed that none of the plants expressing amiR-TSWV-M1 and one-third of the plants expressing amiR-TSWV-M-3 neither showed viral symptoms (Fig. 5D and E) nor accumulated TSWV (Fig. 5F). In contrast, all plants expressing amiRTSWV-M-2, amiR-TSWV-M-4, or amiR-TSWV-M-5 were symptomatic. In some of them, symptoms appeared with a slight delay compared with controls (Fig. 5D and E) and accumulated high levels of TSWV (Fig. 5F). At 20 dpi, five of six plants expressing amiR-TSWV-M-1 and half of the plants expressing amiR-TSWV-M-3 were still symptomless and did not accumulate TSWV (Fig. 5E and F; Table 2).

Finally, all leaves expressing anti-TSWV-S amiRNAs (Fig. 6A) showed a similar number of necrotic lesions compared with controls (Fig. 6B and C). Accordingly, all plants expressing anti-TSWV-S amiRNAs showed symptoms and accumulated TSWV similarly to controls both at 10 and 20 dpi (Fig. 6D, $\mathrm{E}$, and F). Taken together, these results indicate that only a subset of amiRNAs targeting TSWV segments L or M were very active and blocked TSWV systemic infection in $N$. benthamiana, while none of the amiRNAs against fragment $\mathrm{S}$ were effective.

\section{Functional analysis of anti-TSWV syn-tasiRNAs.}

Next, we tested if a syn-tasiRNA construct including various of the previously analyzed amiRNAs could be effective against TSWV PVR. To that purpose, the 35S:syn-tasiR-TSWV construct was generated by introducing four syn-tasiRNA sequences corresponding to the four most effective anti-TSWV amiRNA sequences in the $p M D C 32 B-A t T A S 1 c-B / c$ vector (Fig. 7A) (Carbonell et al. 2014). Similarly, the 35S:syn-tasiR-GUS control construct was generated by introducing four syn-tasiRNA sequences corresponding to amiR-GUS- 1 and amiR-GUS-2 in $p M D C 32 B-A t T A S 1 c-B / c$ (Fig. 7A). The accumulation of syn-

Table 1. Relevant information of selected artificial microRNAs (amiRNAs) targeting Tomato spotted wilt virus (TSWV) segments L, M, or S

\begin{tabular}{|c|c|c|c|c|}
\hline amiRNA name & amiRNA sequence & TP score ${ }^{a}$ & TS entropy ${ }^{b}$ & Target fragment (TS coordinates) \\
\hline amiR-TSWV-L1 & UGCUUAAAAUCGUUGUUACCA & 1 & 0 & $\mathrm{~L}(9-29)$ \\
\hline amiR-TSWV-L2 & UGUCCUGCUAAGAACAUUCCA & 1 & 0.22 & $\mathrm{~L}(7,760-7,780)$ \\
\hline amiR-TSWV-L3 & UCAGAGUGCACAAUCCAUCUU & 1 & 0.70 & $\mathrm{~L}(4,500-4,520)$ \\
\hline amiR-TSWV-L4 & UGGUAUACAAACCUUCUUCAU & 1 & 0.85 & $\mathrm{~L}(4,261-4,281)$ \\
\hline amiR-TSWV-L5 & UGUAAGACGUGAUUGUGUCCU & 1 & 1.17 & $\mathrm{~L}(4,061-4,081)$ \\
\hline amiR-TSWV-M1 & UAUCAGCUCUGGGUGAAUCGG & 1.42 & 0.50 & M (772-792) \\
\hline amiR-TSWV-M2 & UUAAUAGUGAACACUAAGCUC & 1.50 & 1.07 & $\mathrm{M}(3,459-3,479)$ \\
\hline amiR-TSWV-M3 & UUGGUAUAGUGGGGCAUACCG & 1.83 & 0.70 & $\mathrm{M}(508-528)$ \\
\hline amiR-TSWV-M4 & UAGAACUAGUGGUAAAAGCGU & 2 & 0.11 & $\mathrm{M}(4,730-4,750)$ \\
\hline amiR-TSWV-M5 & UAACCUUAAUCCAGACAUCUA & 2 & 0.54 & $\mathrm{M}(4,813-4,833)$ \\
\hline amiR-TSWV-S1 & UUCAGACAGGAUUGGAGCCAA & 1.17 & 0.95 & $\mathrm{~S}(2,739-2,759)$ \\
\hline amiR-TSWV-S2 & UUGGGAGGUAGCUUACCUCUA & 1.50 & 0.76 & $\mathrm{~S}(2,549-2,569)$ \\
\hline amiR-TSWV-S3 & UGUACAGCCAUUCAUGGACAA & 1.50 & 1.01 & $S(662-682)$ \\
\hline amiR-TSWV-S4 & UAAGCCUAUGGAUUACCUCUA & 1.50 & 1.11 & $\mathrm{~S}(2,603-2,623)$ \\
\hline amiR-TSWV-S5 & UCUAAGGUUAAGCUCACUCAC & 2 & 0 & $\mathrm{~S}(2,981-3,001)$ \\
\hline
\end{tabular}

\footnotetext{
a TP score $=$ target prediction score from TargetFinder analysis.

b TS entropy = target site entropy.
} 
tasiRNAs derived from 35S:syn-tasiR-TSWV and 35S:syn-tasiRGUS constructs was analyzed in $N$. benthamiana leaves. For that purpose, each syn-tasiRNA construct was co-agroinfiltrated independently with the $35 S: M I R 173 a$ construct that expresses miR173a, which is required for TAS1c-dependent tasiRNA biogenesis (Montgomery et al. 2008b). Northern blot analysis of RNA preparations obtained 2 dpa from agroinfiltrated leaves confirmed syn-tasiRNA accumulation from both constructs (Supplementary Fig. S4).

As for amiRNAs, the antiviral activity of both syn-tasiRNA constructs was analyzed in six $N$. benthamiana plants by first coagroinfiltrating each syn-tasiRNA construct with $35 S: M I R 173 a$ and, then, inoculating TSWV PVR in the agroinfiltrated leaves 2 days later. As an additional control, 35S:syn-tasiR-TSWV was co-agroinfiltrated with 35S:GUS in the absence of 35SMIR173a. Symptom appearance and ELISA detection of TSWV were surveyed as in the previous amiRNA assays.
Leaves coexpressing the 35S:syn-tasiR-TSWV and 35S:MIR173a constructs did not show necrotic lesions at 5 dpi. In contrast, all leaves coexpressing 35S:syn-tasiR-GUS and 35S:MIR173a or 35S:syn-tasiR-TSWV and 35S:GUS showed a high number of local lesions (Fig. 7B and C). At both 10 and 20 dpi, none of the plants coexpressing syn-tasiR-TSWV and miR173 showed symptoms or accumulated TSWV, in contrast with the rest of TSWV-inoculated controls (Fig. 7D to F). Altogether these results indicate that TSWV-specific syn-tasiRNAs are highly active blocking TSWV infection in $N$. benthamiana and that their activity depends on the coexpression of miR173.

Comparative analysis of anti-TSWV activity of amiRNAs and syn-tasiRNAs against two different TSWV isolates.

Next, we compared the inhibitory effect of amiRNAs and syn-tasiRNAs against two different TSWV isolates, PVR and LL-N.05. To simplify the analysis, we studied the antiviral

\section{TSWV-L segment}
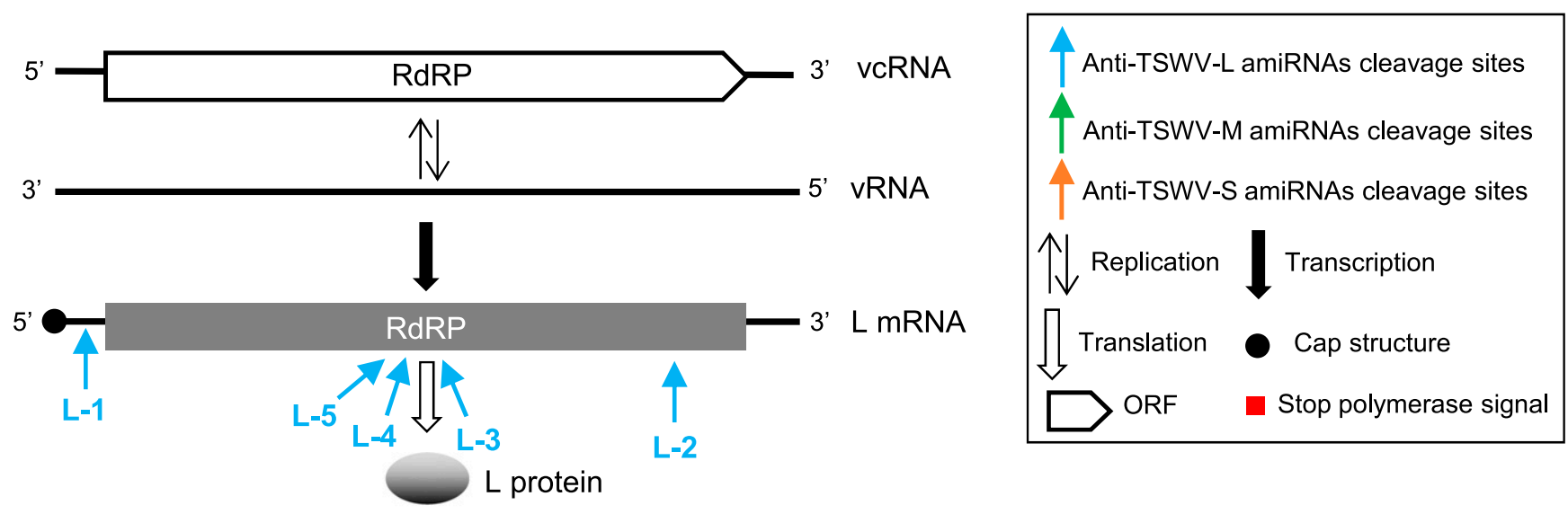

TSWV-M segment

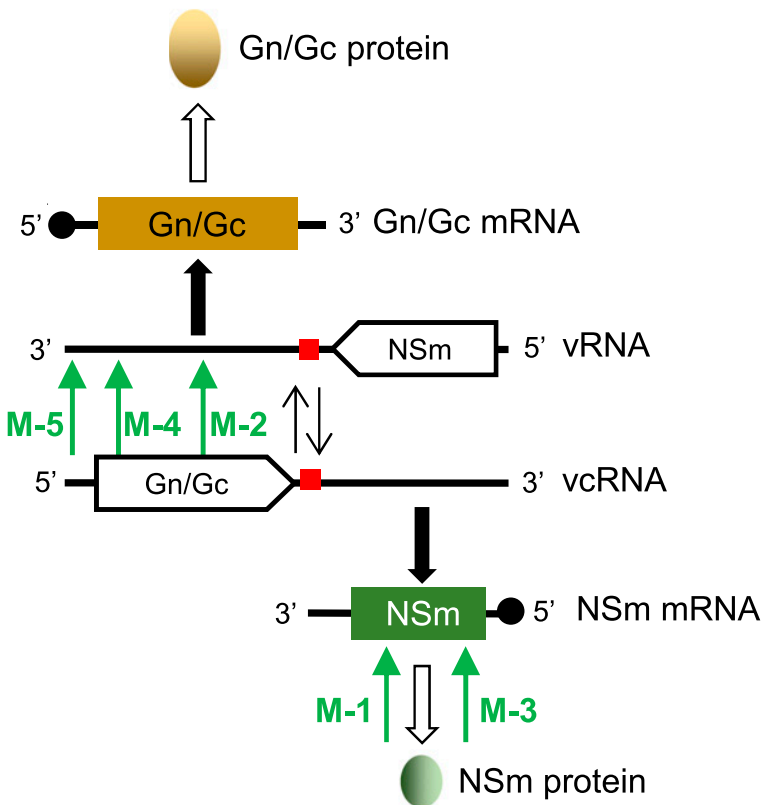

TSWV-S segment
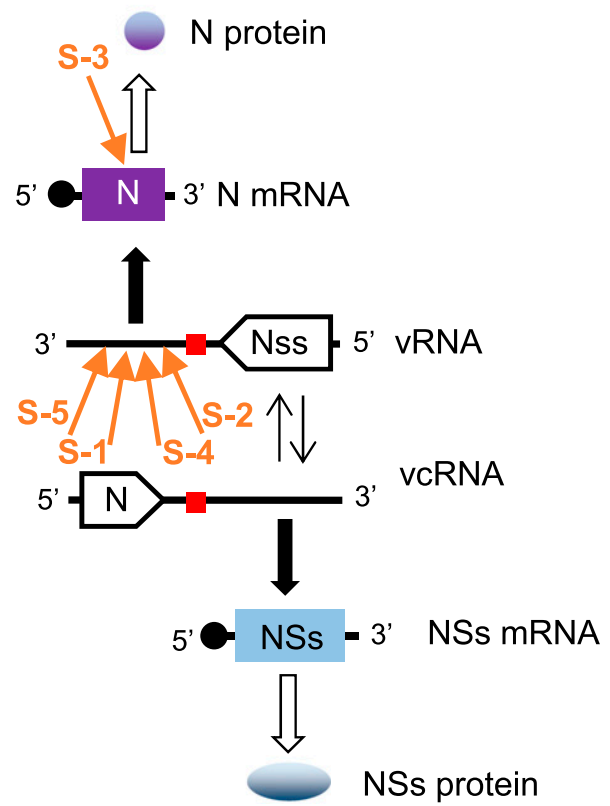

Fig. 3. Localization of artificial microRNA (amiRNA) target sites in Tomato spotted wilt virus (TSWV) genomic and antigenomic RNAs. Approximate amiRNA cleavage site position in $\mathrm{L}, \mathrm{M}$, and $\mathrm{S}$ segments is indicated with arrows. Top right, the meaning of other shapes is indicated in a box. vRNA = viral RNA, vcRNA = viral complementary RNA. 
effects of 35S:amiR-TSWV-L-5, the most effective anti-TSWV amiRNA construct, and 35S:syn-tasiR-TSWV compared with 35S:GUS, when inoculated with the corresponding TSWV isolate. As 35S:MIR173a had to be coexpressed with 35S:syntasiR-TSWV to trigger syn-tasiRNA production, it was also co-agroinfiltrated in samples including 35S:amiR-TSWV-L-5 or
35S:GUS for comparative purposes. Each isolate was inoculated in an independent experiment including 12 plants per block. The antiviral activity of both classes of artificial sRNAs was analyzed as described in the initial amiRNA experiments.

Leaves agroinfiltrated with either 35S:amiR-TSWV-L-5 or 35S:syn-tasiR-TSWV and subsequently inoculated with the
A AtMIR390a-based constructs

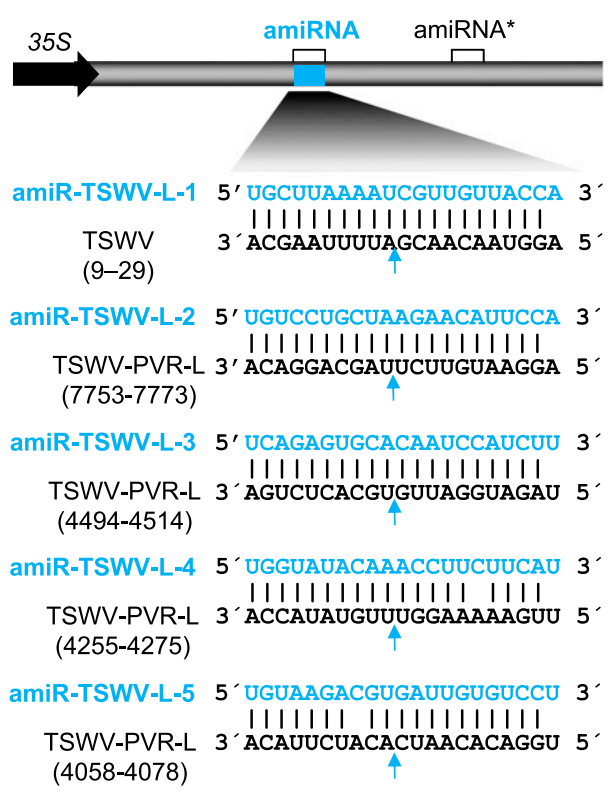

B
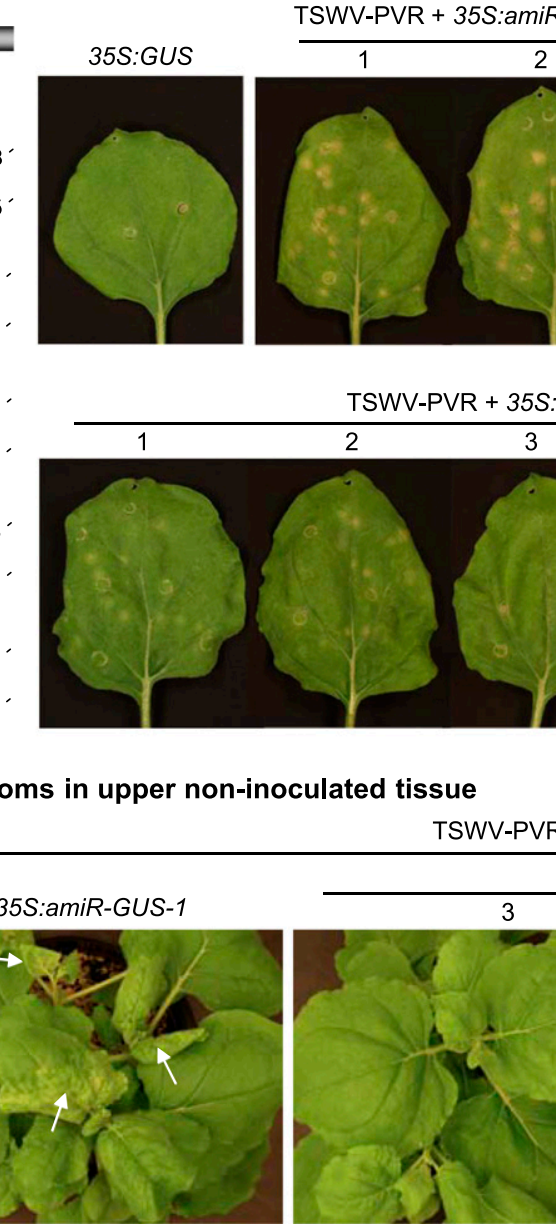

TSWV-PVR
C Analysis of TSWV-induced local lesions in inoculated tissue

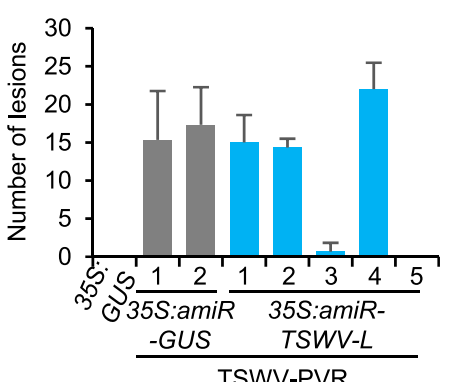

TSWV-PVR
TSWV-PVR + 35S:amiR-GUS

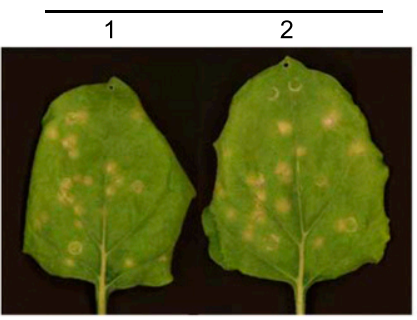

TSWV-PVR + 35S:amiR-TSWV-L

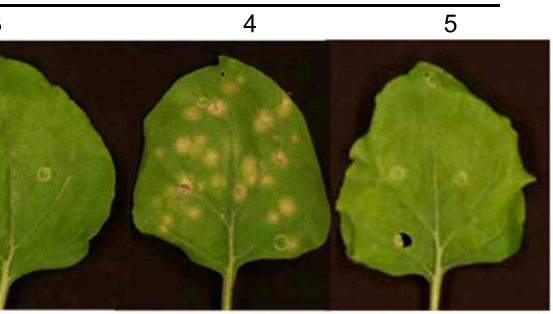

\section{Analysis of TSWV-induced symptoms in upper non-inoculated tissue}

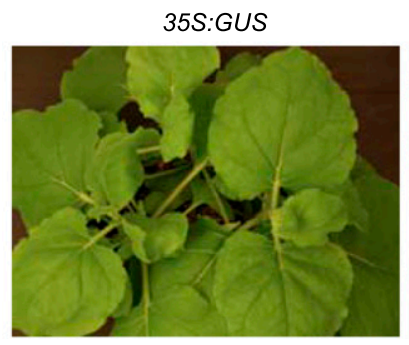

E

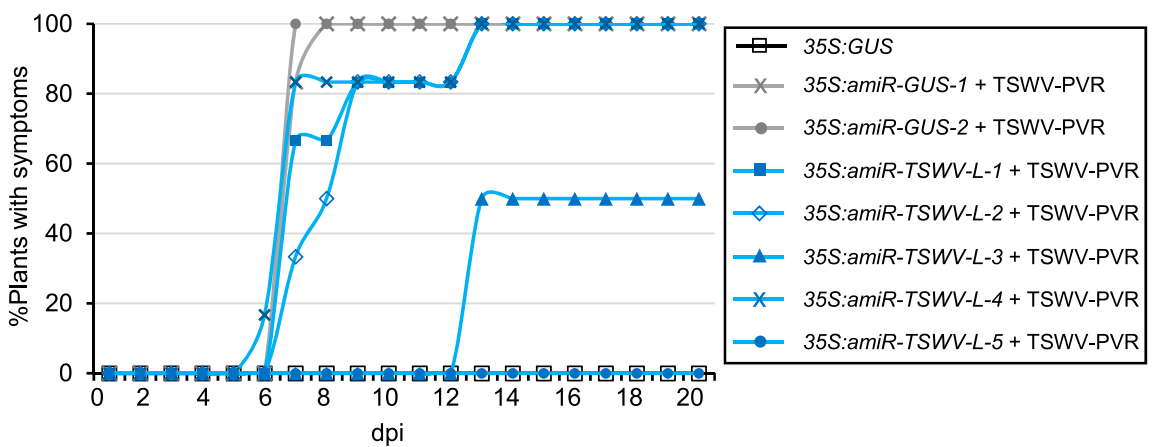

35S:amiR-TSWV-L

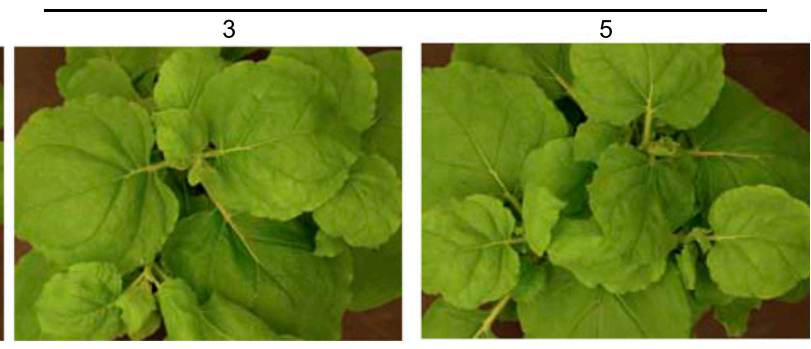

F Analysis of TSWV accumulation in upper non-inoculated tissue

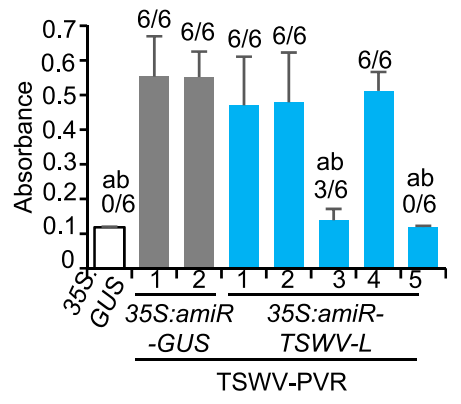

Fig. 4. Analysis of the antiviral activity of several artificial microRNAs (amiRNAs) targeting Tomato spotted wilt virus (TSWV) segment L RNAs. A, Base pairing of amiRNAs and target TSWV PVR RNAs. Anti-TSWV amiRNA and TSWV PVR-L sequences are shown. Coordinates of the complete target site in TSWV PVR-L RNAs are given. The arrows indicate the amiRNA-predicted cleavage site. B, Photos at 7 days postinoculation (dpi) of agroinfiltrated leaves further inoculated with TSWV PVR. C, Bar graph showing the mean number $(n=6)+$ standard deviation (SD) of local lesions in indicated samples at 7 dpi. D, Photos, at $10 \mathrm{dpi}$, of sets of three plants agroinfiltrated and further inoculated with TSWV PVR as indicated. TSWV-induced characteristic symptoms of leaf chlorosis and epinasty are indicated by arrows. E, Two-dimensional line graph showing, for each six-plant set listed in the box, the percentage of symptomatic plants per day for 20 dpi. F, Bar graph representing the mean $(n=6)+$ SD absorbance obtained in double antibody sandwich enzyme-linked immunosorbent assays on indicated samples collected at $10 \mathrm{dpi}$, as an indirect estimate of TSWV accumulation. Bars with the letters a and b are statistically significantly different from that of sample 35S:amiR-GUS-1 + TSWV PVR and 35S:amiR-GUS-2 + TSWV PVR, respectively $(P<0.05$ in pair-wise Student's $t$ test comparisons). 
TSWV PVR isolate showed no or few necrotic lesions, in contrast to leaves coexpressing 35S:GUS (Fig. 8A and B). Analysis of upper noninoculated tissue at 10 dpi showed that none of the plants coexpressing amiR-TSWV-L-5 displayed symptoms, although, in two of them, TSWV was detected by ELISA (Fig. 8C to E). Similarly, only one plant coexpressing
syn-tasiR-TSWV showed symptoms, while two of them accumulated TSWV PVR (Fig. 8C to E). In contrast, all plants coexpressing 35S:GUS showed symptoms and accumulated TSWV PVR (Fig. 8C to E). At $20 \mathrm{dpi}$, two of 12 plants coexpressing 35S:amiR-TSWV-L-5 or 35S:syn-tasiR-TSWV showed symptoms (Fig. 8D).
A

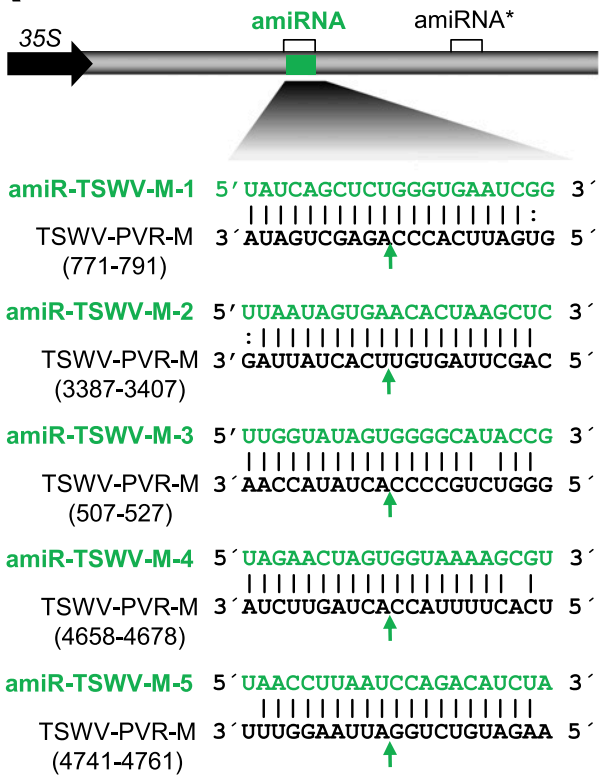

B Analysis of TSWV-induced

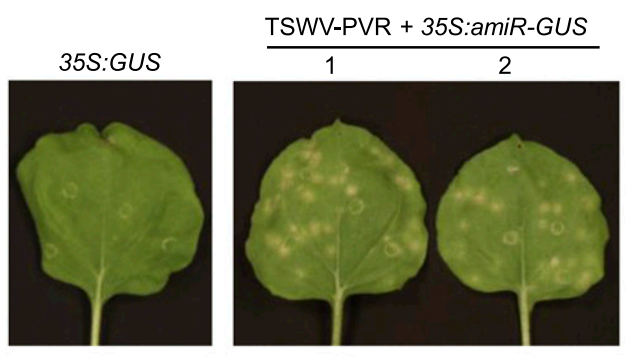

TSWV-PVR+ 35S:amiR-TSWV-M

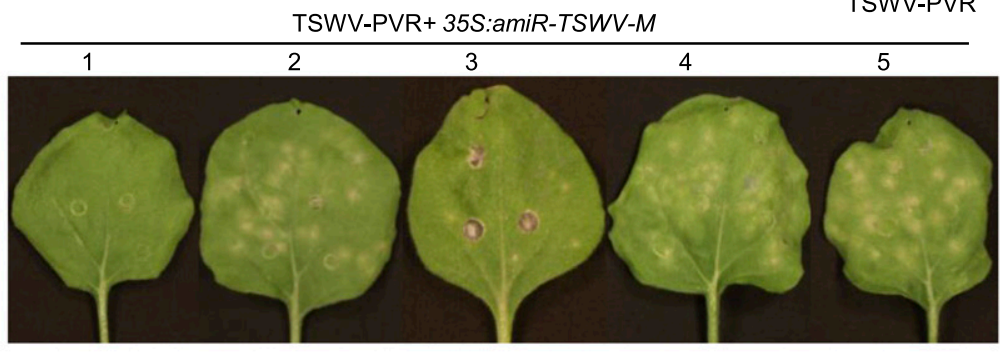
symptoms in inoculated tissue

C Analysis of TSWV-induced local lesions in inoculated tissue

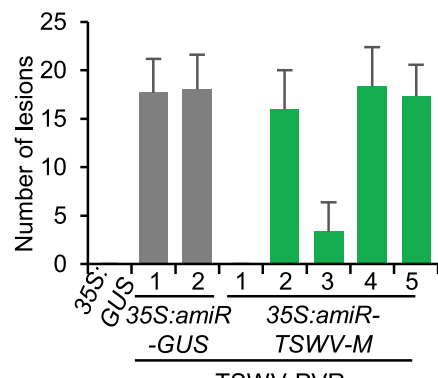

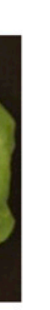

\section{Analysis of TSWV-induced symptoms in upper non-inoculated tissue}

D

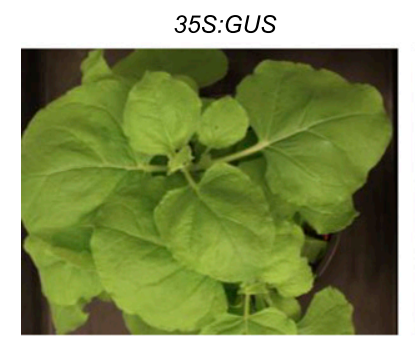

E

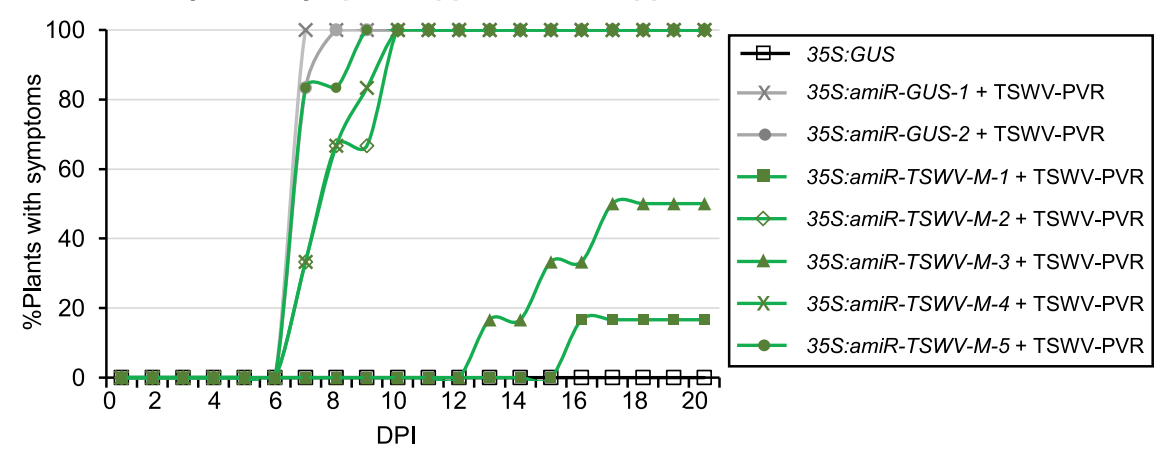

35S:amiR-GUS-1
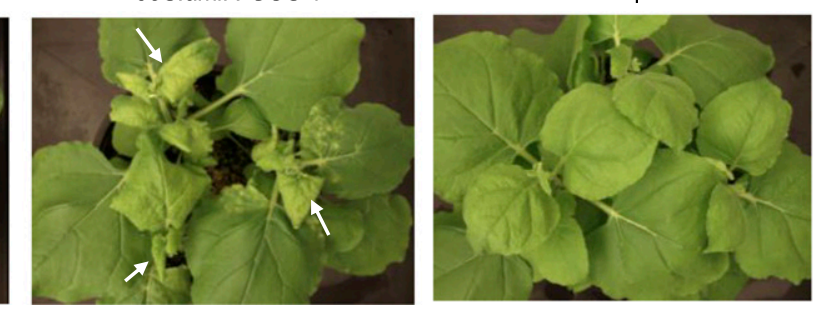

35S:amiR-TSWV-M

$\mathbf{F}$

Analysis of TSWV accumulation in upper non-inoculated tissue

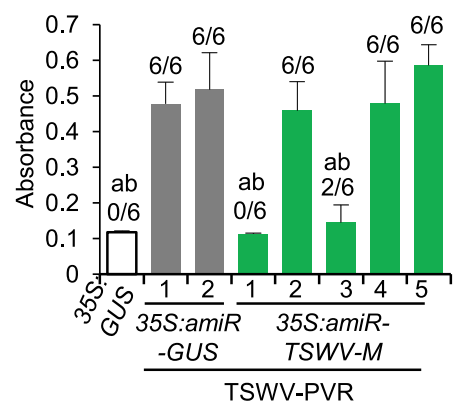

Fig. 5. Analysis of the antiviral activity of several artificial microRNAs (amiRNAs) targeting Tomato spotted wilt virus (TSWV) segment M RNAs. A, Base pairing of amiRNAs and target TSWV PVR RNAs. Anti-TSWV amiRNA and TSWV PVR-M sequences are shown. Coordinates of the complete target site in TSWV PVR-L RNAs are given. The arrows indicate the amiRNA-predicted cleavage site. B, Photos at 7 days postinoculation (dpi) of agroinfiltrated leaves further inoculated with TSWV PVR. C, Bar graph showing the mean number $(n=6)+$ standard deviation (SD) of local lesions in indicated samples at 7 dpi. D, Photos, at $10 \mathrm{dpi}$, of sets of three plants agroinfiltrated and further inoculated with TSWV PVR as indicated. TSWV-induced characteristic symptoms of leaf chlorosis and epinasty are indicated by arrows. E, Two-dimensional line graph showing, for each six-plant set listed in the box, the percentage of symptomatic plants per day for 20 dpi. F, Bar graph representing the mean $(n=6)+$ SD absorbance obtained in double antibody sandwich enzyme-linked immunosorbent assays on indicated samples collected at $10 \mathrm{dpi}$, as an indirect estimate of TSWV accumulation. Bars with the letters a and b are statistically significantly different from that of sample 35S:amiR-GUS-1 + TSWV PVR and 35S:amiR-GUS-2 + TSWV PVR, respectively $(P<0.05$ in pair-wise Student's $t$ test comparisons). 
As described before for TSWV PVR inoculum, an extract of $N$. benthamiana plants infected with TSWV-LL-N.05 was titrated. The dilution assay showed that both the undiluted (ratio 1:20 tissue/buffer) and the 1/10 diluted extracts induced symptoms in all inoculated plants, although these appeared earlier and more homogenously in the case of the undiluted extract (between 6 and $7 \mathrm{dpi}$ ). Therefore, the undiluted extract was used as TSWV-LL-N.05 inoculum. Leaves agroinfiltrated with either 35S:amiR-TSWV-L-5 or 35S:syn-tasiR-TSWV and further inoculated with TSWV-LL-N.05 isolate showed no or few necrotic lesions, in contrast with leaves expressing 35S: GUS (Fig. 9A and B). At $10 \mathrm{dpi}$, none of the plants expressing
A

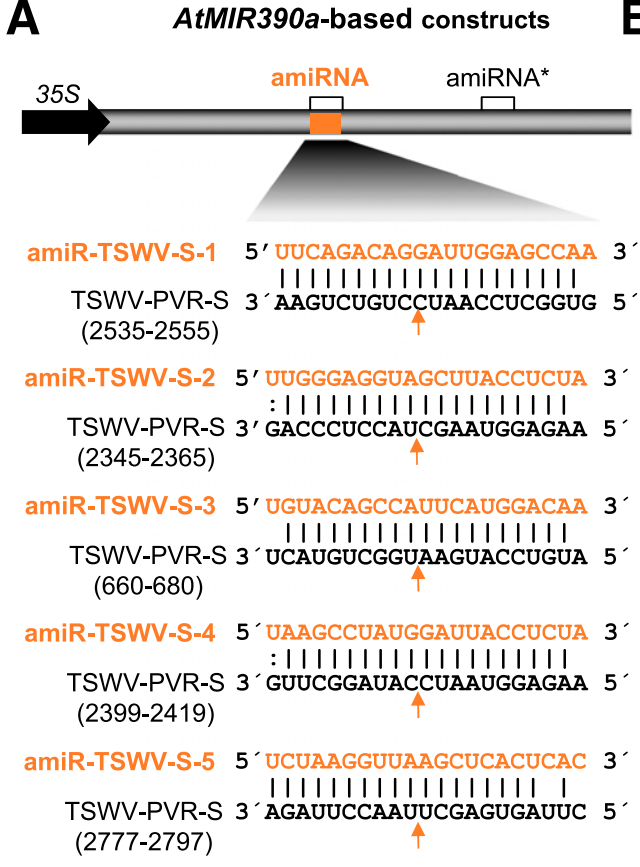

\section{B}
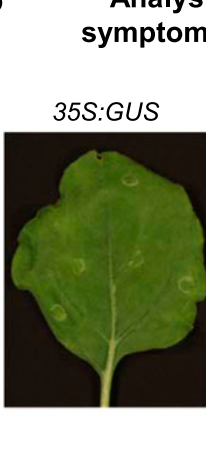

Analysis of TSWV-induced ymptoms in inoculated tissue TSWV-PVR + 35S:amiR-GUS

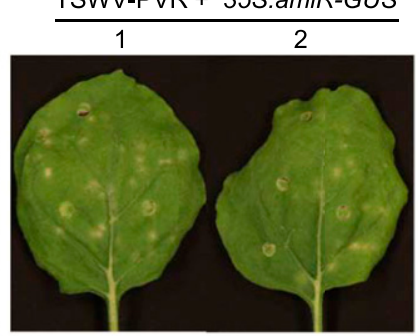

TSWV-PVR + 35S:amiR-TSWV-S

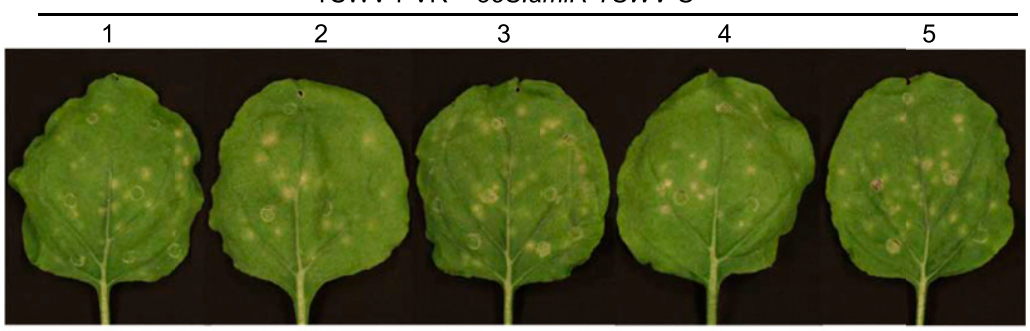

C Analysis of TSWV-induced local lesions in inoculated tissue

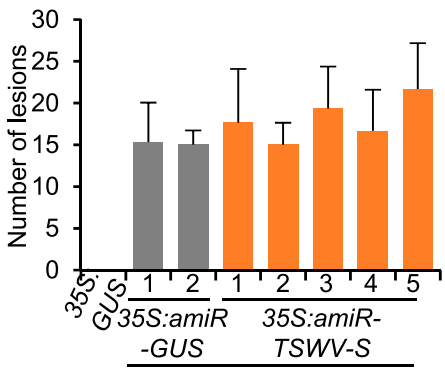
-GUS TSWV-S TSWV-PVR

D Analysis of TSWV-induced symptoms in upper non-inoculated tissue

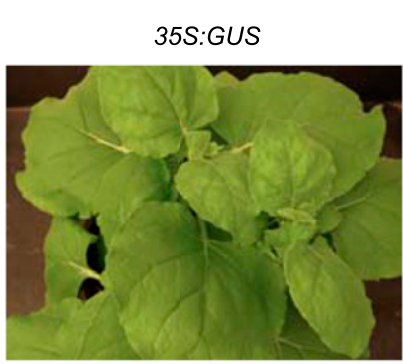

E
Analysis of symptom appearance in upper non-inoculated tissue

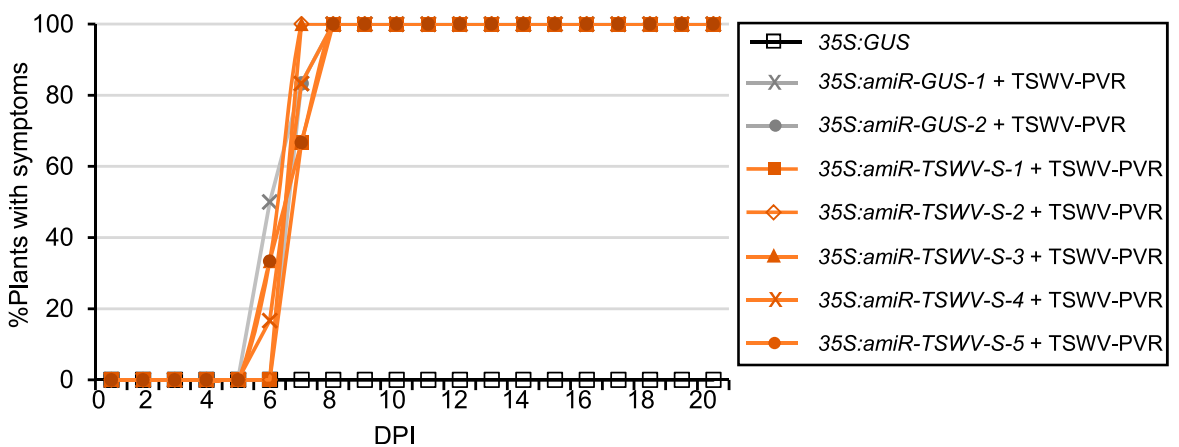

TSWV-PVR

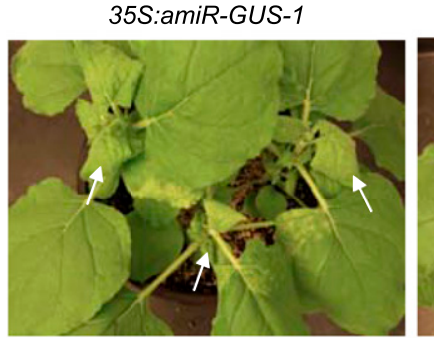

F Analysis of TSWV accumulation in upper non-inoculated tissue

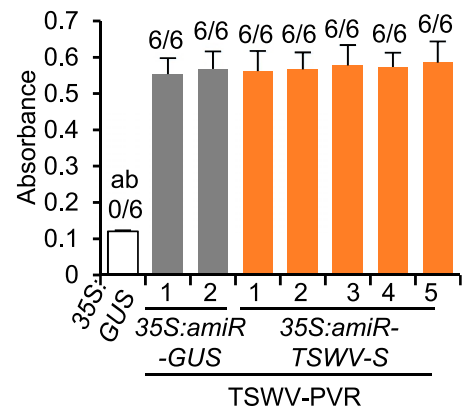

Fig. 6. Analysis of the antiviral activity of several artificial microRNAs (amiRNAs) targeting Tomato spotted wilt virus (TSWV) S segment RNAs. A, Base pairing of amiRNAs and target TSWV PVR RNAs. Anti-TSWV amiRNA and TSWV PVR-S sequences are shown. B, Photos at 7 days postinoculation (dpi) of agroinfiltrated leaves further inoculated with TSWV PVR. C, Bar graph showing the mean number $(n=6)+$ standard deviation (SD) of local lesions in indicated samples at $7 \mathrm{dpi}$. D, Photos, at $10 \mathrm{dpi}$, of sets of three plants agroinfiltrated and further inoculated with TSWV PVR as indicated. TSWV-induced characteristic symptoms of leaf chlorosis and epinasty are indicated by arrows. E, Two-dimensional line graph showing, for each six-plant set listed in the box, the percentage of symptomatic plants per day for 20 dpi. F, Bar graph representing the mean $(n=6)+$ SD absorbance obtained in double antibody sandwich enzyme-linked immunosorbent assays on indicated samples collected at $10 \mathrm{dpi}$, as an indirect estimate of TSWV accumulation. Bars with the letters a and $\mathrm{b}$ are statistically significantly different from that of sample 35S:amiR-GUS-1 + TSWV PVR and 35S:amiR-GUS-2 + TSWV PVR, respectively $(P<0.05$ in pairwise Student's $t$ test comparisons). 
artificial sRNAs showed symptoms (Fig. 9D), although TSWVLL-N.05 was detected by ELISA in two of the plants expressing amiR-TSWV-L-5 and in one plant expressing syntasiR-TSWV (Fig. 9E). At 20 dpi, five of 12 plants expressing amiR-TSWV-L-5 and three of 12 plants expressing syn-tasiRTSWV (Fig. 9D showed symptoms and accumulated TSWVLL-N.05 (Table 1). All combined, these results indicate that both classes of artificial sRNAs are similarly effective against two different TSWV isolates.

\section{DISCUSSION}

Classic RNAi approaches versus artificial sRNAs.

Classic RNAi strategies expressing small interfering RNAs (siRNAs) from transgene-derived double-stranded RNAs (dsRNAs) of viral sequence have been widely used to confer antiviral resistance in plants (Pooggin 2017; Watson et al. 2005). However, these strategies lack high specificity due to the high risk of off-target effects because of the potential accidental targeting of cellular transcripts sharing high sequence complementarity with transgene-derived siRNAs. Moreover, the possibility of recombination between the transgene or transgene-derived transcripts and the genome of related pathogens cannot be discarded. These important limitations were overcome by the development of more recent, potent, and highly precise RNAi technologies based on artificial sRNAs (Carbonell 2017a).

Artificial sRNAs offer a more specific, biosafe, and versatile alternative to classic RNAi approaches for inducing antiviral resistance in plants. First, the shortness of the artificial sRNAs (21 nt) compared with longer RNAi dsRNAs (from 0.3 to several kilobases) facilitates the specificity analysis during the artificial sRNA design in Web-based tools such as WDM3 (Ossowski et al. 2008; Schwab et al. 2010) or P-SAMS (Fahlgren et al. 2016). The specificity analysis requires computational resources and the annotated transcriptome of the species of interest to scrutinize all possible base-pairing interactions between the candidate artificial sRNA and the complete set of host transcripts. Second, the small size of artificial sRNAs increases the biosafety of the transformed crops, as it reduces the possibility of recombination with nontarget viruses (Mitter et al. 2016). And third, amiRNA-mediated resistance has been proved to be more stable than siRNA-based resistance at lower temperatures $\left(15^{\circ} \mathrm{C}\right)$ (Niu et al. 2006). Thus, the amiRNA-mediated approach should

Table 2. Summary of results obtained from symptom and double antibody sandwich enzyme-linked immunosorbent assay (DAS-ELISA) analyses in all bioassays

\begin{tabular}{|c|c|c|c|c|}
\hline \multirow[b]{2}{*}{ Figure/sample } & \multicolumn{2}{|c|}{10 days postinoculation } & \multicolumn{2}{|c|}{20 days postinoculation } \\
\hline & $\begin{array}{c}\text { Symptomatic/ } \\
\text { total }\end{array}$ & $\begin{array}{l}\text { DAS-ELISA } \\
\text { positive/total }\end{array}$ & $\begin{array}{c}\text { Symptomatic/ } \\
\text { total }\end{array}$ & $\begin{array}{l}\text { DAS-ELISA } \\
\text { positive/total }\end{array}$ \\
\hline \multicolumn{5}{|l|}{ Figure 4} \\
\hline 35S:GUS & $0 / 6$ & $0 / 6$ & $0 / 6$ & $0 / 6$ \\
\hline 35S:amiR-GUS-1 + TSWV PVR & $6 / 6$ & $6 / 6$ & $6 / 6$ & $6 / 6$ \\
\hline 35S:amiR-GUS-2 + TSWV PVR & $6 / 6$ & $6 / 6$ & $6 / 6$ & $6 / 6$ \\
\hline 35S:amiR-TSWV-L-1 + TSWV PVR & $6 / 6$ & $6 / 6$ & $6 / 6$ & $6 / 6$ \\
\hline 35S:amiR-TSWV-L-2 + TSWV PVR & $6 / 6$ & $6 / 6$ & $6 / 6$ & $6 / 6$ \\
\hline 35S:amiR-TSWV-L-3 + TSWV PVR & $6 / 6$ & $6 / 6$ & $6 / 6$ & $6 / 6$ \\
\hline 35S:amiR-TSWV-L-4 + TSWV PVR & $3 / 6$ & $3 / 6$ & $3 / 6$ & $3 / 6$ \\
\hline 35S:amiR-TSWV-L-5 + TSWV PVR & $0 / 6$ & $0 / 6$ & $0 / 6$ & $0 / 6$ \\
\hline \multicolumn{5}{|l|}{ Figure 5} \\
\hline 35S:GUS & $0 / 6$ & $0 / 6$ & $0 / 6$ & $0 / 6$ \\
\hline 35S:amiR-GUS-1 + TSWV PVR & $6 / 6$ & $6 / 6$ & $6 / 6$ & $6 / 6$ \\
\hline 35S:amiR-GUS-2 + TSWV PVR & $6 / 6$ & $6 / 6$ & $6 / 6$ & $6 / 6$ \\
\hline 35S:amiR-TSWV-M-1 + TSWV PVR & $0 / 6$ & $0 / 6$ & $1 / 6$ & $1 / 6$ \\
\hline 35S:amiR-TSWV-M-2 + TSWV PVR & $6 / 6$ & $6 / 6$ & $6 / 6$ & $6 / 6$ \\
\hline 35S:amiR-TSWV-M-3 + TSWV PVR & $2 / 6$ & $2 / 6$ & $3 / 6$ & $3 / 6$ \\
\hline 35S:amiR-TSWV-M-4 + TSWV PVR & $6 / 6$ & $6 / 6$ & $6 / 6$ & $6 / 6$ \\
\hline 35S:amiR-TSWV-M-5 + TSWV PVR & $6 / 6$ & $6 / 6$ & $6 / 6$ & $6 / 6$ \\
\hline \multicolumn{5}{|l|}{ Figure 6} \\
\hline $35 S: G U S$ & $0 / 6$ & $0 / 6$ & $0 / 6$ & $0 / 6$ \\
\hline 35S:amiR-GUS-1 + TSWV PVR & $6 / 6$ & $6 / 6$ & $6 / 6$ & $6 / 6$ \\
\hline 35S:amiR-GUS-2 + TSWV PVR & $6 / 6$ & $6 / 6$ & $6 / 6$ & $6 / 6$ \\
\hline 35S:amiR-TSWV-S-1 + TSWV PVR & $6 / 6$ & $6 / 6$ & $6 / 6$ & $6 / 6$ \\
\hline 35S:amiR-TSWV-S-2 + TSWV PVR & $6 / 6$ & $6 / 6$ & $6 / 6$ & $6 / 6$ \\
\hline 35S:amiR-TSWV-S-3 + TSWV PVR & $6 / 6$ & $6 / 6$ & $6 / 6$ & $6 / 6$ \\
\hline 35S:amiR-TSWV-S-4 + TSWV PVR & $6 / 6$ & $6 / 6$ & $6 / 6$ & $6 / 6$ \\
\hline 35S:amiR-TSWV-S-5 + TSWV PVR & $6 / 6$ & $6 / 6$ & $6 / 6$ & $6 / 6$ \\
\hline \multicolumn{5}{|l|}{ Figure 7} \\
\hline 35S:GUS & $0 / 6$ & $0 / 6$ & $0 / 6$ & $0 / 6$ \\
\hline 35S:syn-tasiR-GUS + 35S:MIR173 + TSWV PVR & $6 / 6$ & $6 / 6$ & $6 / 6$ & $6 / 6$ \\
\hline 35S:syn-tasiR-TSWV + 35S:GUS + TSWV PVR & $6 / 6$ & $6 / 6$ & $6 / 6$ & $6 / 6$ \\
\hline 35S:syn-tasiR-TSWV + 35S:MIR173 + TSWV PVR & $0 / 6$ & $0 / 6$ & $0 / 6$ & $0 / 6$ \\
\hline \multicolumn{5}{|l|}{ Figure 8} \\
\hline 35S:GUS & $0 / 12$ & $0 / 12$ & $0 / 12$ & $0 / 12$ \\
\hline 35S:GUS + 35S:MIR173 + TSWV PVR & $12 / 12$ & $12 / 12$ & $12 / 12$ & $12 / 12$ \\
\hline 35S:amiR-TSWV-L-5 + 35S:MIR173 + TSWV PVR & $0 / 12$ & $2 / 12$ & $2 / 12$ & $2 / 12$ \\
\hline 35S:syn-tasiR-TSWV + 35S:MIR173 + TSWV PVR & $1 / 12$ & $2 / 12$ & $2 / 12$ & $2 / 12$ \\
\hline \multicolumn{5}{|l|}{ Figure 9} \\
\hline 35S:GUS & $0 / 12$ & $0 / 12$ & $0 / 12$ & $0 / 12$ \\
\hline 35S:GUS + 35S:MIR173 + TSWV-LL-N.05 & $12 / 12$ & $12 / 12$ & $12 / 12$ & $12 / 12$ \\
\hline 35S:amiR-TSWV-L-5 + 35S:MIR173 + TSWV-LL-N.05 & $0 / 12$ & $2 / 12$ & $5 / 12$ & $5 / 12$ \\
\hline 35S:syn-tasiR-TSWV + 35S:MIR173 + TSWV-LL-N.05 & $0 / 12$ & $1 / 12$ & $3 / 12$ & $3 / 12$ \\
\hline
\end{tabular}


A

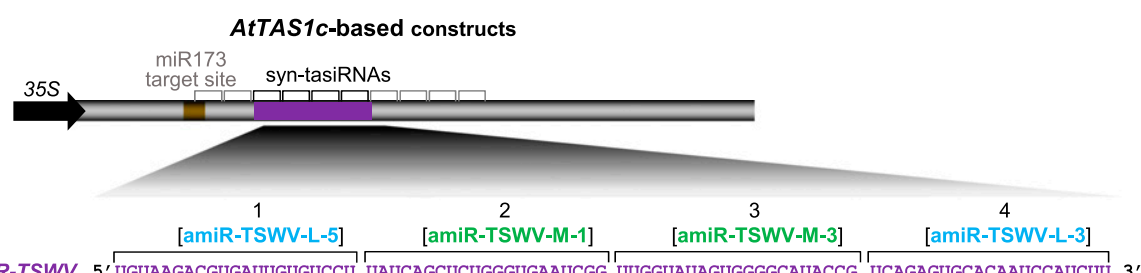

syn-tasiR-TSWV 5 'UGUAAGACGUGAUUGUGUCCU UAUCAGCUCUGGGUGAAUCGG UUGGUAUAGUGGGGCAUACCG UCAGAGUGCACAAUCCAUCUU 3 TSWV-PVR 3' ACAUUCUACACUAACACAGGU AUAGUCGAGACCACUUAGUG AACCAUAUCACCCCGUCUGG

$\begin{array}{cccc}\text { TSWV-L } & \text { TSWV-M } & \text { TSWV-M } & \text { TWV-L } \\ (4058-4078) & (771-791) & (507-527) & (4494-4514) \\ 1 & 2 & 3 & 4\end{array}$

$\begin{array}{cccc}1 & 2 & 3 & 4 \\ \text { (amiR-GUS-1) } & \text { (amiR-GUS-2) } & \text { (amiR-GUS-1) } & \text { (amiR-GUS-2) }\end{array}$

syn-tasiR-GUS 5' UAUUGACCCACACUUUGCCGA UAACCUUCACCCGGUGCCAC UAUUGACCCACACUUUGCCGA UAACCUUCACCCGGUGCCAC 3' GUS 3' AUAACUGGGUGGAACGGCA AUUGGAAGUGGCCACGGUC AUACUGGGUGUGAACGGCA AUUGGAGUGGGCAACGGC 5

(280-300)

(722-742)

B

Analysis of TSWV-induced symptoms in inoculated tissue TSWV-PVR

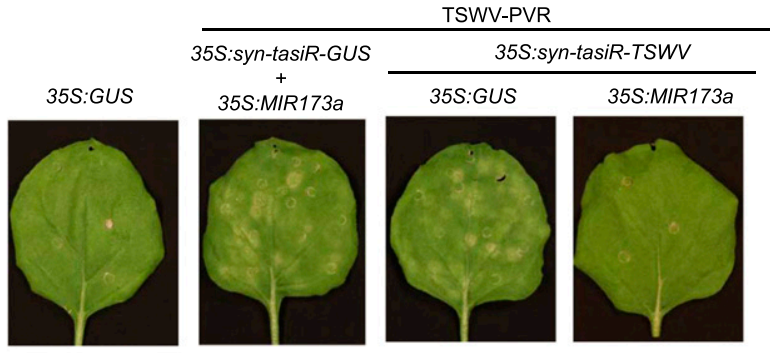

(280-300)

(722-742)

C Analysis of TSWV-induced local lesions in inoculated tissue

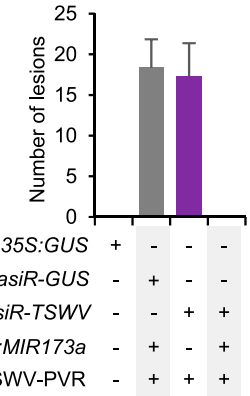

TSWV-PVR - + + +

D Analysis of TSWV-induced symptoms in upper non-inoculated tissue

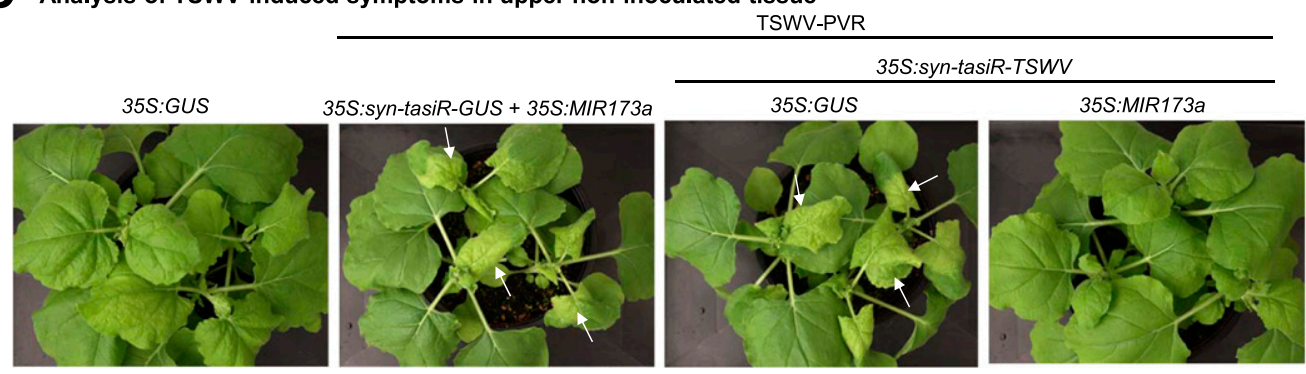

E Analysis of symptom appearance in upper non-inoculated tissue

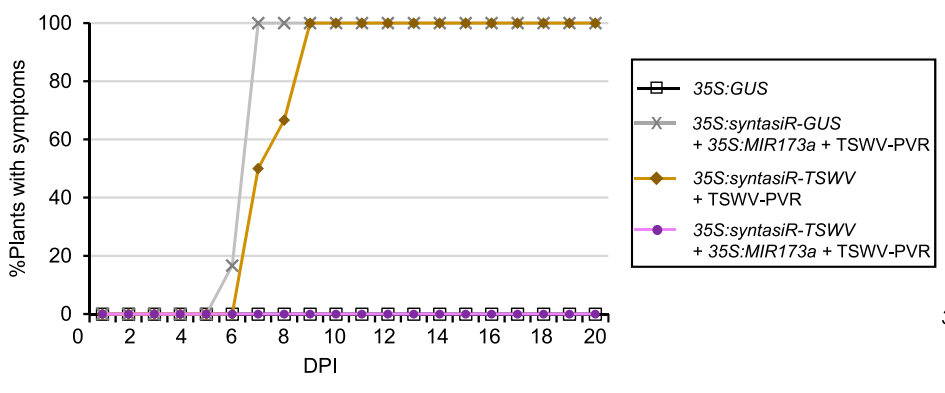

F Analysis of TSWV accumulation in upper non-inoculated tissue

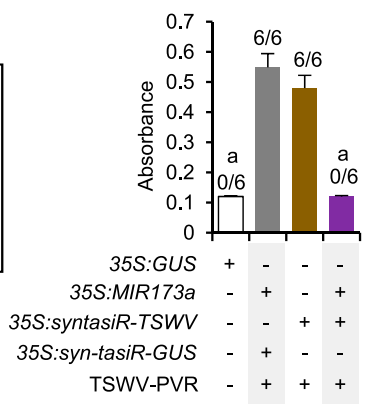

Fig. 7. Analysis of the antiviral activity of synthetic trans-acting small interfering RNAs (syn-tasiRNAs) targeting Tomato spotted wilt virus (TSWV) RNAs. A, Organization of syn-tasiRNA constructs. tasiRNA, and syn-tasiRNA positions are indicated. Base pairing of syn-tasiRNAs and target TSWV PVR RNAs is shown. B, Photos at 7 days postinoculation (dpi) of agroinfiltrated leaves further inoculated with TSWV PVR. C, Bar graph showing the mean number $(n=6)+$ standard deviation (SD) of local lesions in indicated samples at 7 dpi. D, Photos, at 10 dpi, of sets of three plants agroinfiltrated and further inoculated with TSWV PVR as indicated. TSWV-induced characteristic symptoms of leaf chlorosis and epinasty are indicated by arrows. E, Two-dimensional line graph showing, for each six-plant set listed in the box, the percentage of symptomatic plants per day for $20 \mathrm{dpi}$. F, Bar graph representing the mean $(n=6)+\mathrm{SD}$ absorbance obtained in double antibody sandwich enzyme-linked immunosorbent assays on indicated samples collected at 10 dpi, as an indirect estimate of TSWV accumulation. Bars with the letter a are statistically significantly different from that of sample 35S:syn-tasiR-GUS + 35S:MIR173a + TSWV PVR $(P<$ 0.05 in pair-wise Student's $t$ test comparisons). 
have a broader applicability for engineering antiviral resistance in crop plants. Whether syn-tasiRNA-mediated resistance is also stable at this lower temperature needs to be proved.

A fast-forward methodology for the identification of highly active artificial sRNA sequences.

The efficacy of a given artificial sRNA depends on multiple factors, such as the degree of base-pairing between the artificial sRNA and the target RNA or the accessibility of the target site among others. It is known that artificial sRNA efficacy positively correlates with the degree of base-pairing between the
sRNA and the target RNA (Liu et al. 2014). Accordingly, automated tools for artificial sRNA design such as WMD3 (Ossowski et al. 2008) or P-SAMS (Fahlgren et al. 2016) typically optimize for efficacy by designing sRNAs with high sequence complementarity with the target RNA. Other factors influencing artificial sRNA efficacy, such as target site accessibility, are much more difficult to predict and, therefore, are not taken into account during the design process (Fahlgren et al. 2016; Ossowski et al. 2008). The difficulty to predict if a particular artificial sRNA will be highly active in vivo makes it crucial to conduct a preliminary functional screen of multiple

\section{A Analysis of TSWV-induced symptoms in inoculated tissue}

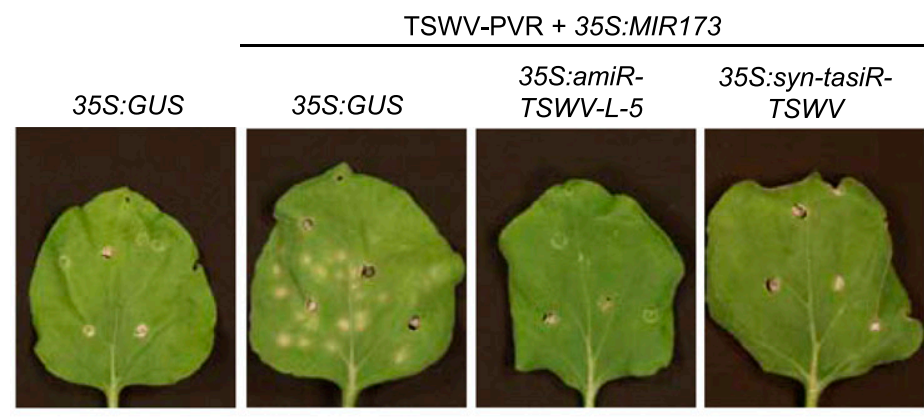

\section{B Analysis of TSWV-induced} local lesions in inoculated tissue

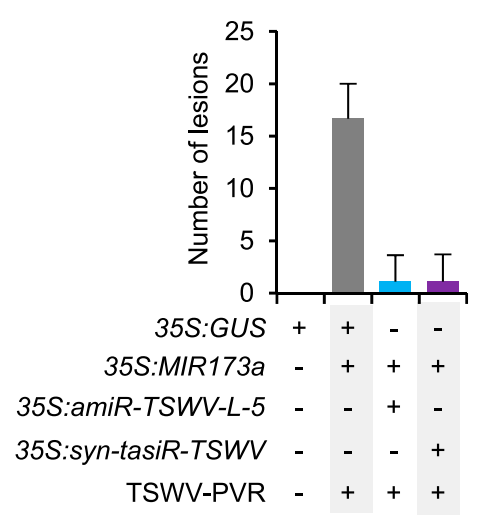

\section{Analysis of TSWV-induced symptoms in upper non-inoculated tissue}

TSWV-PVR + 35S:MIR173
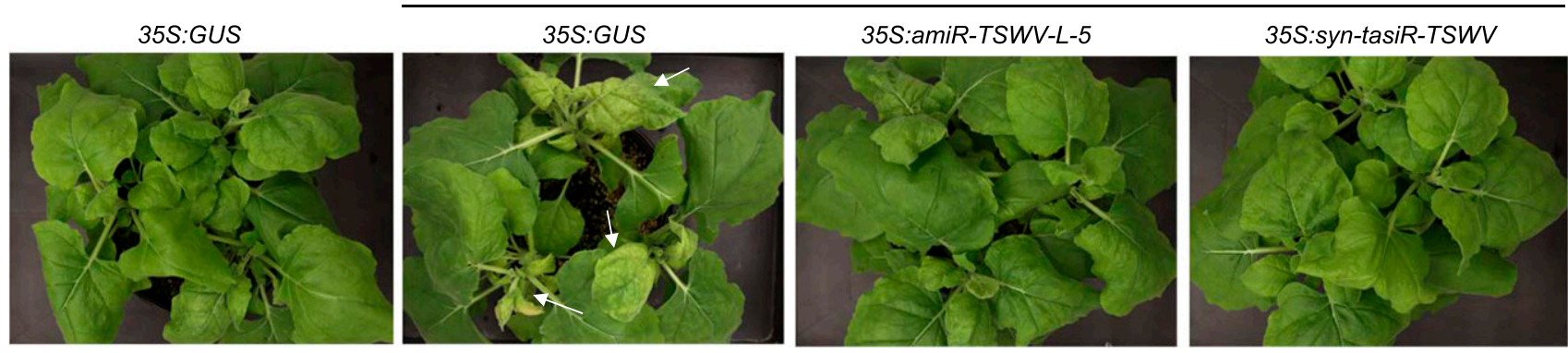

D

Analysis of symptom appearance in upper non-inoculated tissue

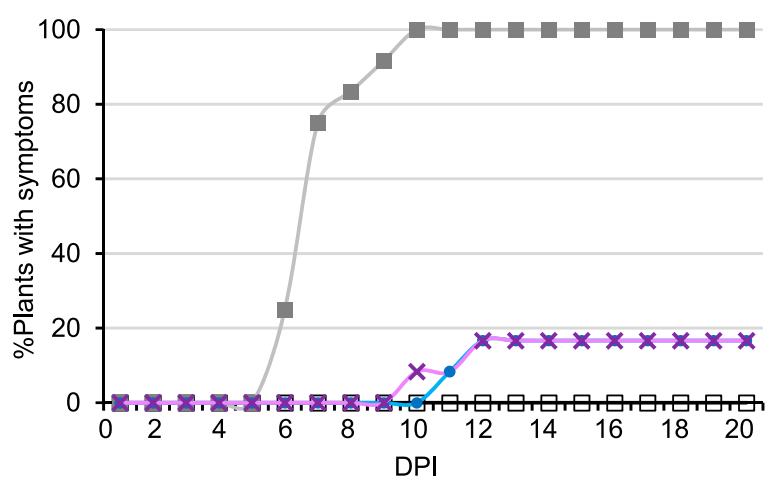

E Analysis of TSWV accumulation in upper non-inoculated tissue

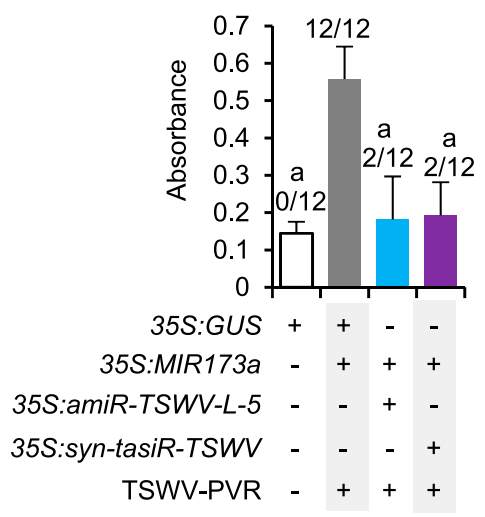

Fig. 8. Comparative analysis of the antiviral activity of artificial microRNAs (amiRNAs) and synthetic trans-acting small interfering RNAs (syn-tasiRNAs) against Tomato spotted wilt virus (TSWV) PVR isolate. A, Photos at 7 days postinoculation (dpi) of agroinfiltrated leaves further inoculated with TSWV PVR. B, Bar graph showing the mean number $(n=6)+$ standard deviation (SD) of local lesions in indicated samples at 7 dpi. C, Photos, at 10 dpi, of sets of three plants agroinfiltrated and further inoculated with TSWV PVR as indicated. TSWV-induced characteristic symptoms of leaf chlorosis and epinasty are indicated by arrows. D, Two-dimensional line graph showing, for each six-plant set listed in the box, the percentage of symptomatic plants per day for 20 dpi. E, Bar graph representing the mean $(n=6)+$ SD absorbance obtained in double antibody sandwich enzyme-linked immunosorbent assays on indicated samples collected at $10 \mathrm{dpi}$, as an indirect estimate of TSWV accumulation. Bars with the letter a are statistically significantly different from that of sample 35S:GUS + 35S:MIR173a + TSWV PVR ( $P<0.05$ in pair-wise Student's $t$ test comparisons). 
artificial sRNAs to identify those with the highest activity before starting stable plant transformation. Pertinent to this context, it has been shown that highly active amiRNAs transiently expressed in $N$. benthamiana remain highly effective when expressed in A. thaliana transgenic plants (Yu and Pilot 2014), thus further supporting the usefulness of preliminary functional screens.

Here, we coupled the recently described high-throughput methods for generating artificial sRNA constructs for plants to a screening system based on agroinfiltration in $N$. benthamiana, to identify optimal artificial sRNAs against an important viral pathogen of solanaceous plants, such as TSWV. Importantly, the whole process including the rational design using the $\mathrm{P}$ SAMS tool (Fahlgren et al. 2016), the high-throughput generation of plant artificial sRNA constructs using the new generation of $\mathrm{B} / \mathrm{c}$ vectors optimized for one-step cloning and high expression of artificial sRNA sequences (Carbonell et al. 2014, 2015), and plant agroinfiltration is completed in just a week (Carbonell, in press). Moreover, this methodology should be of broad interest, as it can be applied to search for resistance to any of the large list of viruses that infect $N$. benthamiana (Goodin et al. 2008).
A Analysis of TSWV-induced symptoms in inoculated tissue
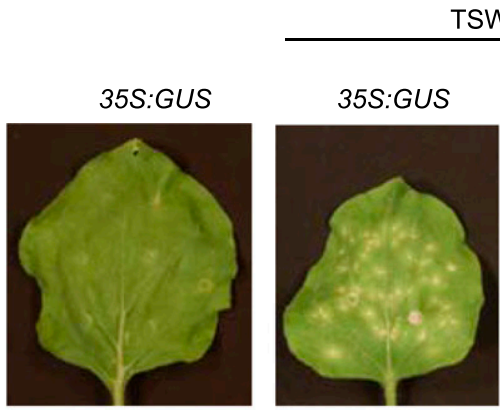

TSWV-LL-N-0.5 + 35S:MIR173a

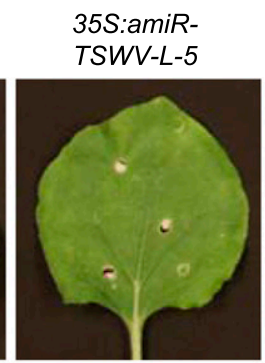

35S:syn-tasiR-

TSWV

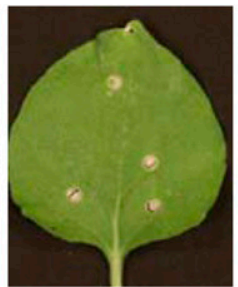

Analysis of TSWV-induced symptoms in upper non-inoculated tissue

\section{B Analysis of TSWV-induced local lesions in inoculated tissue}

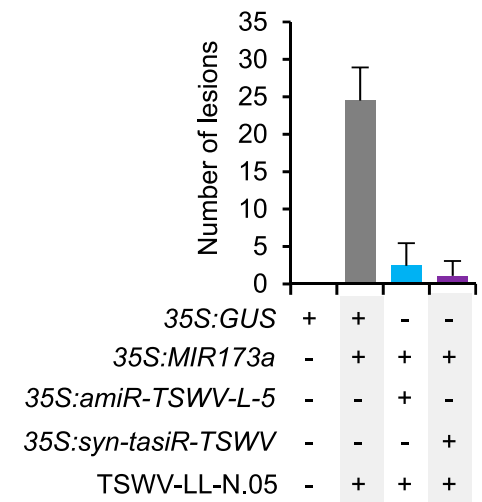

C

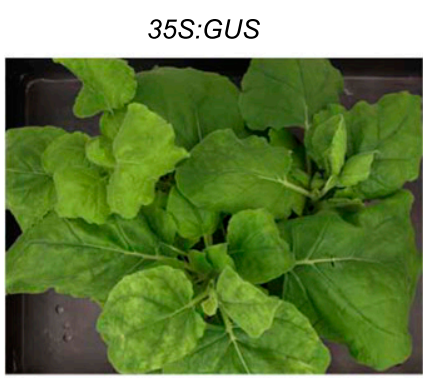

TSWV-LL-N.05 + 35S:MIR173a

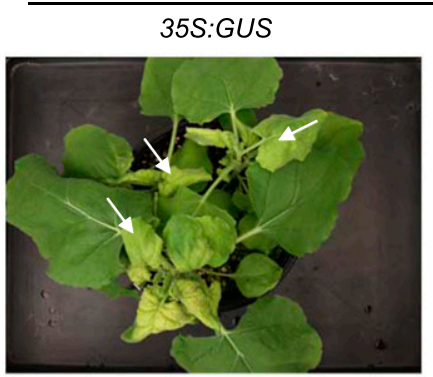

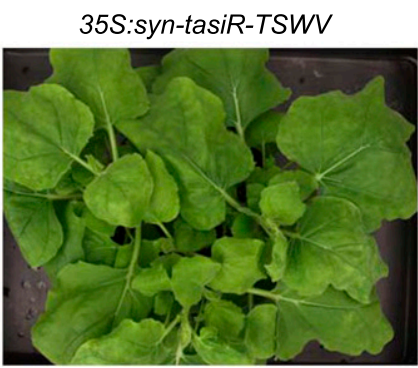

\section{Analysis of symptom appearance in upper non-inoculated tissue}

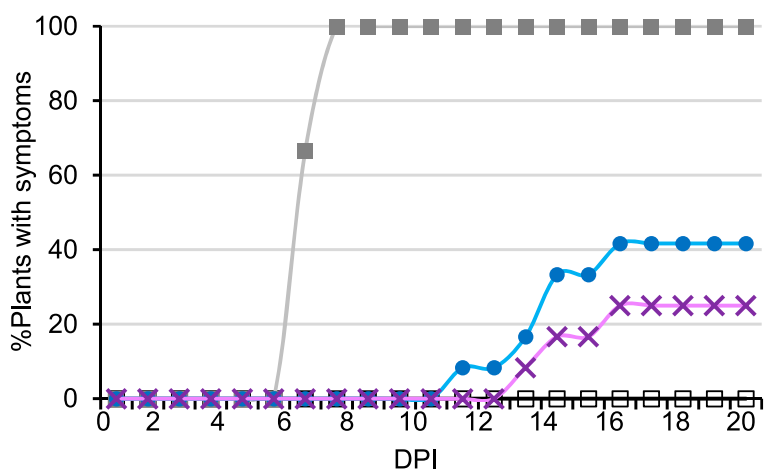

E Analysis of TSWV accumulation in upper non-inoculated tissue

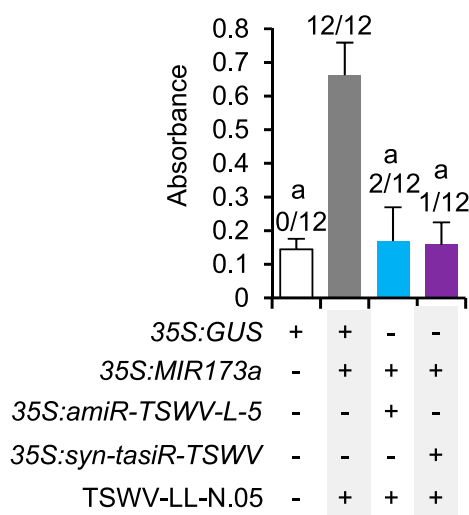

Fig. 9. Comparative analysis of the antiviral activity of artificial microRNAs (amiRNAs) and synthetic trans-acting small interfering RNAs (syn-tasiRNAs) against Tomato spotted wilt virus (TSWV) LL-N.05 isolate. A, Photos at 7 days postinoculation (dpi) of agroinfiltrated leaves further inoculated with TSWV PVR. B, Bar graph showing the mean number $(n=6)+$ standard deviation $(\mathrm{SD})$ of local lesions in indicated samples at 7 dpi. C, Photos, at $10 \mathrm{dpi}$, of sets of three plants agroinfiltrated and further inoculated with TSWV PVR as indicated. TSWV-induced characteristic symptoms of leaf chlorosis and epinasty are indicated by arrows. D, Two-dimensional line graph showing, for each six-plant set listed in the box, the percentage of symptomatic plants per day for 20 dpi. E, Bar graph representing the mean $(n=6)+$ SD absorbance obtained in double antibody sandwich enzyme-linked immunosorbent assays on indicated samples collected at $10 \mathrm{dpi}$, as an indirect estimate of TSWV accumulation. Bars with the letter a are statistically significantly different from that of sample 35S:GUS + 35S:MIR173a + TSWV-LL-N.05 ( $P<0.05$ in pair-wise Student's $t$ test comparisons). 
Different efficacies of anti-TSWV amiRNAs.

The amiRNA functional screen showed that only a subset of the amiRNAs tested were highly active against TSWV. In particular, only two of five amiRNAs targeting the L segment and two of five amiRNAs targeting the $\mathrm{M}$ segment were active, while none of the five amiRNAs targeting segment $\mathrm{S}$ were functional. Why were only a subset of amiRNAs active and others not? First, it could be argued that more active amiRNAs may accumulate to higher levels. In our study, more active amiRNAs (amiR-TSWV-L-3, amiR-TSWV-L-5, amiR-TSWVM1, and amiR-TSWV-M3) are among the more highly accumulated amiRNA species analyzed at 2 dpa. However, other highly accumulated amiRNAs, such as amiR-TSWV-M-4 and, especially, amiR-TSWV-S-1, are inactive, suggesting that a high accumulation is probably required but not sufficient for an amiRNA to be active. It is also possible that different amiRNAs have different degradation rates over time, with amiRNA instability negatively affecting amiRNA activity. Second, regarding the degree of base-pairing between amiRNAs and target sites in TSWV PVR RNAs, in our work, all productive and unproductive amiRNA/target RNA interactions present only one or two missmatches within specific amiRNA regions (position 1 or 14 to 21) known to have a modest effect on sRNA efficacy based on studies on Arabidopsis thaliana miRNAs (Fahlgren and Carrington 2010). Therefore, the degree of base-pairing between amiRNA and target RNA does not explain, by itself, the differences in efficacy observed between amiRNAs in our study. Moreover, the hybrid formed by the most active amiRNA (amiRNA-TSWV-L-5) and its target RNA in TSWV PVR presents two mismatches, one at position 21 that should have a modest effect and another at position 8 (Fig. 4A), which is included in a specific sRNA region (positions 2 to 14) in which mismatches are known to drastically decrease sRNA activity (Fahlgren and Carrington 2010). This result suggests that other unconventional but productive miRNA/target interactions may occur, as the one formed by an mRNA encoding the blue copperbinding protein and miR398 in A. thaliana, which includes a bulge of six nucleotides opposite to the $5^{\prime}$ region of the miRNA (Brousse et al. 2014). And third, it is possible that other factors more difficult to predict, such as target site accessibility, affect more significantly the efficacy of each amiRNA, as previously proposed (Carbonell and Daròs 2017). Indeed, it seems that accessibility in natural plant miRNA target sites is influenced by the local mRNA secondary structure and by the putative positioning of various mRNA binding proteins (Liu et al. 2014). For all these reasons, it is not simple to explain why a specific amiRNA is functional and another is not, as highlighted in a previous work describing that only two amiRNAs targeting the TSWV $N$ gene were active while another two amiRNAs targeting the TSWV NSs gene were not (Mitter et al. 2016). Again, these considerations strongly support the need for preliminary screening of multiple artificial sRNA sequences to identify those with high activity.

\section{amiRNA versus syn-tasiRNA.}

Since their first use to confer antiviral resistance (Niu et al. 2006), amiRNAs have been widely employed to protect plants from viruses with high specificity mainly in transgenic plants (Liu et al. 2017). Because antiviral amiRNAs only target a single site in viral RNAs, the amiRNA-induced resistance is typically overcome because of mutations in the amiRNA target site in the viral progeny (Lafforgue et al. 2011; Lin et al. 2009). To increase the durability of the amiRNA protection, multiple amiRNAs have been expressed from constructs including several precursors in tandem or a single polycistronic precursor (Fahim et al. 2012; Kis et al. 2016; Kung et al. 2012; Lafforgue et al. 2013). Syn-tasiRNAs have more recently emerged as an alternative to amiRNA to induce specific, potent antiviral resistance in plants. The main advantage of the syn-tasiRNA approach is that multiple artificial sRNAs can be multiplexed in a single construct, thus allowing for the simultaneous targeting of multiple target sites within a viral RNA or of multiple sequence-unrelated viruses. This should increase the durability of the syn-tasiRNA-mediated resistance, as the possibility that the virus mutates all target sites to break the resistance appears unlikely (Carbonell et al. 2016). Syn-tasiRNAs have been recently shown to confer resistance against two different viruses (Chen et al. 2016) as well as against viroids (Carbonell and Daròs 2017), although the durability of the protection was not analyzed. Here, the comparative analysis between the most efficient amiRNA (amiR-TSWV-L-5) and the syn-tasiRNAs showed that both classes of artificial sRNAs were highly effective against two different TSWV isolates in N. benthamiana.

\section{Conclusion.}

This work describes the successful application of a fastforward methodology for the identification of highly effective artificial sRNA sequences to suppress TSWV, an important viral pathogen causing dramatic crop losses worldwide. Our results show that both amiRNAs and syn-tasiRNAs, transientlyexpressed in $N$. benthamiana, are highly effective against two different TSWV isolates. Future work is necessary to confirm that both classes of artificial sRNAs can induce high levels of durable anti-TSWV resistance when stably expressed in transformed tomato plants. In any case, the efficient methodology described here should be of broad interest, as it could be applied for the quick identification of artificial sRNA sequences inducing resistance to any of the multiple viruses infecting $N$. benthamiana. More generally, the application of such methodology should definitely accelerate the generation of plants with enhanced, durable antiviral resistance to ensure food security in the near future.

\section{MATERIALS AND METHODS}

\section{TSWV isolates.}

Complete sequences corresponding to TSWV segments L, M and $S$ (29, 65, and 61 respectively) were collected from the NCBI Nucleotide database. FASTA sequences of all segments for all isolates are included in Supplementary Text S1. The most relevant information regarding TSWV isolates used in P-SAMS-based amiRNA designs or in infection assays is included in Supplementary Table S1.

\section{Entropy analyses.}

Clustal Omega with default parameters was used to independently align all the sequences of each segment. The Shannon entropy value of each position was calculated using the equation

$$
\begin{aligned}
\mathrm{E}=\{ & {[\log 2(\text { freq } \mathrm{A}) \times \text { freqA }]+[\log 2(\text { freqC }) \times \text { freqC }] } \\
& +[\log 2(\text { freq }) \times \text { freqG }]+[\log 2(\text { freq } \mathrm{T}) \times \text { freq } \mathrm{T}]\} \\
& \times(-1),
\end{aligned}
$$

where "E" is "Entropy", and "freqA", "freqC", "freqG" and "freqT" are the frequency of adenine, cytosine, guanine, and thymine, respectively, at this particular position (with gaps being considered as elements of variation). Finally, target site entropy was calculated as the sum of individual entropies of each target site position.

\section{Artificial sRNA design.}

An updated version of the P-SAMS script returning unlimited optimal results was used to retrieve the complete list of optimal amiRNAs targeting TSWV L, M, or S segments. Input 
sequences used in amiRNA designs are included in Supplementary Text S2. The off-targeting filtering in S. lycopersicum transcriptome iTAGv2.3 was enabled in all amiRNA designs. TargetFinder (Fahlgren and Carrington 2010) script was run to confirm that selected amiRNAs do not target significantly $N$. benthamiana transcriptome v5.1 (Nakasugi et al. 2014) (Supplementary Dataset S4).

\section{DNA constructs.}

Artificial sRNA constructs were generated following the methodology previously described (Carbonell et al. 2014) and using the oligonucleotides output by P-SAMS. AmiRNA constructs 35S:amiR-TSWV-L-1, 35S:amiR-TSWV-L-2, 35S:amiRTSWV-L-3, 35S:amiR-TSWV-L-4, 35S:amiR-TSWV-L-5, 35S: amiR-TSWV-M-1, 35S:amiR-TSWV-M-2, 35S:amiR-TSWVM-3, 35S:amiR-TSWV-M-4, 35S:amiR-TSWV-M-5, 35S:amiRTSWV-S-1, 35S:amiR-TSWV-S-2, 35S:amiR-TSWV-S-3, 35S: amiR-TSWV-S-4, and 35S:amiR-TSWV-S-5 were obtained by ligating annealed oligo pairs D2127/D2128, D2129/D2130, D2131/D2132, D2133/D2134, D2135/D2136, D2137/D2138, D2139/D2140, D2141/D2142, D2143/D2144, D2145/D2146, D2147/D2148, D2149/D2150 D2151/D2152, D2153/D2154, and D2155/D2156, respectively, into pMDC32B-AtMIR390a-B/c (Addgene plasmid 51776) (Carbonell et al. 2014). Syn-tasiRNA constructs 35S:syn-tasiR-TSWV and 35S:syn-tasiR-GUS were obtained by ligating annealed oligo pairs AC-45/AC-46 and AC47/AC-48, respectively, into $p M D C 32 B-A t T A S 1 c-B / c$ (Addgene plasmid 51773) (Carbonell et al. 2014). DNA oligonucleotides used to generate the artificial sRNA constructs are listed in Supplementary Table S3.

35S:amiR-GUS-1 and 35S:amiR-GUS-2, 35S:GUS and 35S: MIR173a constructs were described previously (Carbonell and Daròs 2017; Montgomery et al. 2008a).

\section{Plant bioassays and TSWV inoculation.}

$N$. benthamiana plants were grown in a growth chamber at $25^{\circ} \mathrm{C}$ with a 12 -h light and 12 -h dark photoperiod. For each TSWV isolate, a crude extract from $N$. benthamiana-infected tissue was used as inoculum and was applied to the whole surface of the third true leaf of 3-week-old plants as described (Carbonell and Daròs, in press). In artificial sRNA-based functional assays, this third leaf was agroinfiltrated 2 days before with cultures of Agrobacterium tumefaciens GV3101, as described (Carbonell et al. 2012; Cuperus et al. 2010).

\section{RNA gel blot assays.}

Total RNA from $N$. benthamiana leaves was isolated using TRIzol reagent (Thermo Fisher Scientific) followed by chloroform extraction. RNA was precipitated with an equal volume of isopropanol for $20 \mathrm{~min}$. Triplicate samples from two infiltrated leaves each were analyzed. RNA gel-blot assays were done as described (Cuperus et al. 2010; Montgomery et al. 2008b). Briefly, $20 \mu \mathrm{g}$ of total RNA were resolved by denaturing polyacrylamide gel electrophoresis in $17 \%$ polyacrylamide gels containing $0.5 \times$ Tris-borate-EDTA and $7 \mathrm{M}$ urea anwered transferred to a positively-charged nylon membrane. Northern blot hybridizations were done at $40^{\circ} \mathrm{C}$ in the presence of PerfectHyb Plus hybridization buffer (Sigma-Aldrich) with DNA probes end-labeled using $\left[\gamma_{-}{ }^{32} \mathrm{P}\right]$ ATP and T4 polynucleotide kinase (Thermo Fisher Scientific). A Fujifilm FLA-5100 imaging system was used to measure blot hybridization signals (photostimulated luminescence). DNA oligonucleotides used as probes are listed in Supplementary Table S4.

\section{Double antibody sandwich (DAS)-ELISA assays.}

TSWV accumulation in extracts from apical leaves collected at 10 or 20 dpi were analyzed by DAS-ELISA, using the TSWV
Complete kit (Bioreba) essentially as indicated by the manufacturer. Briefly, samples were homogenized in extraction buffer at a 1:50 dilution and two technical replicates of $0.1 \mathrm{ml}$ of extract were analyzed. The absorbance of each sample was measured at $405 \mathrm{~nm}$ using a model 550 microplate reader (Bio-Rad). Samples were considered to be infected (DASELISA-positive) when absorbance was higher than three times the average absorbance of the samples from non-TSWVinoculated controls. The absorbance values were used as an indirect estimate of the viral accumulation as reported previously (Soler-Aleixandre et al. 2007).

\section{ACKNOWLEDGMENTS}

We thank V. Aragonés for invaluable technical assistance and J. Forment for helping with the entropy analyses.

\section{LITERATURE CITED}

Brousse, C., Liu, Q., Beauclair, L., Deremetz, A., Axtell, M. J., and Bouché, N. 2014. A non-canonical plant microRNA target site. Nucleic Acids Res. 42:5270-5279.

Bucher, E., Lohuis, D., van Poppel, P. M., Geerts-Dimitriadou, C., Goldbach, R., and Prins, M. 2006. Multiple virus resistance at a high frequency using a single transgene construct. J. Gen. Virol. 87:3697-3701.

Carbonell, A. Design and high-throughput generation of artificial small RNA constructs for plants. Methods Mol. Biol. In press.

Carbonell, A. 2017a. Artificial small RNA-based strategies for effective and specific gene silencing in plants. Pages 110-127 in: Plant Gene Silencing: Mechanisms and Applications. T. Dalmay, ed. CABI Publishing, Wallingford, U.K

Carbonell, A. 2017b. Plant ARGONAUTEs: Features, functions, and unknowns. Methods Mol. Biol. 1640:1-21.

Carbonell, A., Carrington, J.C., and Daròs, J.A. 2016. Fast-forward generation of effective artificial small RNAs for enhanced antiviral defense in plants. RNA Dis. 3:e1130.

Carbonell, A., and Daròs, J. A. 2017. Artificial microRNAs and synthetic trans-acting small interfering RNAs interfere with viroid infection. Mol. Plant Pathol. 18:746-753.

Carbonell, A., and Daròs, J. A. Design, synthesis and functional analysis of highly specific artificial small RNAs with antiviral activity in plants. Methods Mol. Biol. In press.

Carbonell, A., Fahlgren, N., Garcia-Ruiz, H., Gilbert, K. B., Montgomery, T. A., Nguyen, T., Cuperus, J. T., and Carrington, J. C. 2012. Functional analysis of three Arabidopsis ARGONAUTES using slicer-defective mutants. Plant Cell 24:3613-3629.

Carbonell, A., Fahlgren, N., Mitchell, S., Cox, K. L., Jr., Reilly, K. C., Mockler, T. C., and Carrington, J. C. 2015. Highly specific gene silencing in a monocot species by artificial microRNAs derived from chimeric miRNA precursors. Plant J. 82:1061-1075.

Carbonell, A., Takeda, A., Fahlgren, N., Johnson, S. C., Cuperus, J. T., and Carrington, J. C. 2014. New generation of artificial microRNA and synthetic trans-acting small interfering RNA vectors for efficient gene silencing in Arabidopsis. Plant Physiol. 165:15-29.

Chen, L., Cheng, X., Cai, J., Zhan, L., Wu, X., Liu, Q., and Wu, X. 2016. Multiple virus resistance using artificial trans-acting siRNAs. J. Virol. Methods 228:16-20.

Cuperus, J. T., Carbonell, A., Fahlgren, N., Garcia-Ruiz, H., Burke, R. T., Takeda, A., Sullivan, C. M., Gilbert, S. D., Montgomery, T. A., and Carrington, J. C. 2010. Unique functionality of 22-nt miRNAs in triggering RDR6-dependent siRNA biogenesis from target transcripts in Arabidopsis. Nat. Struct. Mol. Biol. 17:997-1003.

de la Luz Gutiérrez-Nava, M., Aukerman, M. J., Sakai, H., Tingey, S. V., and Williams, R. W. 2008. Artificial trans-acting siRNAs confer consistent and effective gene silencing. Plant Physiol. 147:543-551.

Debreczeni, D. E., López, C., Aramburu, J., Darós, J. A., Soler, S., Galipienso, L., Falk, B. W., and Rubio, L. 2015. Complete sequence of three different biotypes of tomato spotted wilt virus (wild type, tomato Sw-5 resistance-breaking and pepper Tsw resistance-breaking) from Spain. Arch. Virol. 160:2117-2123.

Fahim, M., Millar, A. A., Wood, C. C., and Larkin, P. J. 2012. Resistance to Wheat streak mosaic virus generated by expression of an artificial polycistronic microRNA in wheat. Plant Biotechnol. J. 10:150-163.

Fahlgren, N., and Carrington, J. C. 2010. miRNA target prediction in plants. Methods Mol. Biol. 592:51-57. 
Fahlgren, N., Hill, S. T., Carrington, J. C., and Carbonell, A. 2016. PSAMS: A web site for plant artificial microRNA and synthetic transacting small interfering RNA design. Bioinformatics 32:157-158.

Goodin, M. M., Zaitlin, D., Naidu, R. A., and Lommel, S. A. 2008 Nicotiana benthamiana: Its history and future as a model for plantpathogen interactions. Mol. Plant-Microbe Interact. 21:1015-1026.

Jan, F.-J., Fagoaga, C., Pang, S.-Z., and Gonsalves, D. 2000. A minimum length of $\mathrm{N}$ gene sequence in transgenic plants is required for RNAmediated tospovirus resistance. J. Gen. Virol. 81:235-242.

Kis, A., Tholt, G., Ivanics, M., Várallyay, É., Jenes, B., and Havelda, Z 2016. Polycistronic artificial miRNA-mediated resistance to Wheat dwarf virus in barley is highly efficient at low temperature. Mol. Plant Pathol. 17:427-437.

Kormlink, R. 2011. The molecular biology of tospoviruses and resistance strategies. Pages 163-191 in: Bunyaviridae: Molecular and Cellular Biology. R. M. Elliot, and A. Plyusin, eds. Plenum Press, New York.

Kung, Y. J., Lin, S. S., Huang, Y. L., Chen, T. C., Harish, S. S., Chua, N. H., and Yeh, S. D. 2012. Multiple artificial microRNAs targeting conserved motifs of the replicase gene confer robust transgenic resistance to negativesense single-stranded RNA plant virus. Mol. Plant Pathol. 13:303-317.

Lafforgue, G., Martínez, F., Niu, Q. W., Chua, N. H., Daròs, J. A., and Elena, S. F. 2013. Improving the effectiveness of artificial microRNA (amiR)-mediated resistance against Turnip Mosaic Virus by combining two amiRs or by targeting highly conserved viral genomic regions. J. Virol. 87:8254-8256.

Lafforgue, G., Martínez, F., Sardanyés, J., de la Iglesia, F., Niu, Q. W., Lin, S. S., Solé, R. V., Chua, N. H., Daròs, J. A., and Elena, S. F. 2011. Tempo and mode of plant RNA virus escape from RNA interference-mediated resistance. J. Virol. 85:9686-9695.

Lin, S. S., Wu, H. W., Elena, S. F., Chen, K. C., Niu, Q. W., Yeh, S. D., Chen, C. C., and Chua, N. H. 2009. Molecular evolution of a viral noncoding sequence under the selective pressure of amiRNA-mediated silencing. PLoS Pathog. 5:e1000312.

Liu, Q., Wang, F., and Axtell, M. J. 2014. Analysis of complementarity requirements for plant microRNA targeting using a Nicotiana benthamiana quantitative transient assay. Plant Cell 26:741-753.

Liu, S. R., Zhou, J. J., Hu, C. G., Wei, C. L., and Zhang, J. Z. 2017. MicroRNA-mediated gene silencing in plant defense and viral counterdefense. Front. Microbiol. 8:1801.

MacKenzie, D. J., and Ellis, P. J. 1992. Resistance to tomato spotted wilt virus infection in transgenic tobacco expressing the viral nucleocapsid gene. Mol. Plant-Microbe Interact. 5:34-40.

Mitter, N., Zhai, Y., Bai, A. X., Chua, K., Eid, S., Constantin, M., Mitchell, R., and Pappu, H. R. 2016. Evaluation and identification of candidate genes for artificial microRNA-mediated resistance to tomato spotted wilt virus. Virus Res. 211:151-158.

Montgomery, T. A., Howell, M. D., Cuperus, J. T., Li, D., Hansen, J. E., Alexander, A. L., Chapman, E. J., Fahlgren, N., Allen, E., and Carrington, J. C. 2008a. Specificity of ARGONAUTE7-miR390 interaction and dual functionality in TAS3 trans-acting siRNA formation. Cell 133:128-141.

Montgomery, T. A., Yoo, S. J., Fahlgren, N., Gilbert, S. D., Howell, M. D. Sullivan, C. M., Alexander, A., Nguyen, G., Allen, E., Ahn, J. H., and Carrington, J. C. 2008b. AGO1-miR173 complex initiates phased siRNA formation in plants. Proc. Natl. Acad. Sci. U.S.A. 105:20055-20062.

Nakasugi, K., Crowhurst, R., Bally, J., and Waterhouse, P. 2014. Combining transcriptome assemblies from multiple de novo assemblers in the allotetraploid plant Nicotiana benthamiana. PLoS One 9:e91776.

Niu, Q. W., Lin, S. S., Reyes, J. L., Chen, K. C., Wu, H. W., Yeh, S. D., and Chua, N. H. 2006. Expression of artificial microRNAs in transgenic
Arabidopsis thaliana confers virus resistance. Nat. Biotechnol. 24: 1420-1428.

Ossowski, S., Schwab, R., and Weigel, D. 2008. Gene silencing in plants using artificial microRNAs and other small RNAs. Plant J. 53:674-690.

Peng, J. C., Chen, T. C., Raja, J. A., Yang, C. F., Chien, W. C., Lin, C. H., Liu, F. L., Wu, H. W., and Yeh, S. D. 2014. Broad-spectrum transgenic resistance against distinct tospovirus species at the genus level. PLoS One 9:e96073.

Plyusnin, A., Beaty, B., Elliot, R., Goldbach, R., Kormelink, R., Lundkvist, A., Schmaljohn, C., and Tesh, R. 2012. Bunyaviridae. Pages 725-741 in: Virus taxonomy: ninth report of the International Commitee on Taxonomy of Viruses. A. M. Q. King, M. J. Adams, E. B. Carsyens, and E. J. Lefkowitz, eds. Elsevier Academic Press, London.

Pooggin, M. M. 2017. RNAi-mediated resistance to viruses: A critical assessment of methodologies. Curr. Opin. Virol. 26:28-35.

Prins, M., Resende, R. O., Anker, C., van Schepen, A., de Haan, P., and Goldbach, R. 1996. Engineered RNA-mediated resistance to tomato spotted wilt virus is sequence specific. Mol. Plant-Microbe Interact. 9:416-418.

Scholthof, K. B., Adkins, S., Czosnek, H., Palukaitis, P., Jacquot, E., Hohn, T., Hohn, B., Saunders, K., Candresse, T., Ahlquist, P., Hemenway, C., and Foster, G. D. 2011. Top 10 plant viruses in molecular plant pathology. Mol. Plant Pathol. 12:938-954.

Schwab, R., Ossowski, S., Riester, M., Warthmann, N., and Weigel, D. 2006. Highly specific gene silencing by artificial microRNAs in Arabidopsis. Plant Cell 18:1121-1133.

Schwab, R., Ossowski, S., Warthmann, N., and Weigel, D. 2010. Directed gene silencing with artificial microRNAs. Methods Mol. Biol. 592:71-88.

Shannon, C. E. 1997. The mathematical theory of communication. 1963. MD Comput. 14:306-317.

Sherwood, J.L., German, T.L., Moyer, J.W., and Ullman, D. E. 2003. Tomato spotted wilt. The Plant Health Instructor, American Phytopathological Society, St. Paul, MN, U.S.A.

Soler-Aleixandre, S., López, C., Cebolla-Cornejo, J., and Nuez, F. 2007. Sources of resistance to Pepino mosaic virus (PepMV) in tomato. HortScience 42:40-45.

Sonoda, S., and Tsumuki, H. 2004. Analysis of RNA-mediated virus resistance by NSs and NSm gene sequences from Tomato spotted wilt virus. Plant Sci. 166:771-778.

Turina, M., Kormelink, R., and Resende, R. O. 2016. Resistance to Tospoviruses in vegetable crops: Epidemiological and molecular aspects. Annu. Rev. Phytopathol. 54:347-371.

Watson, J. M., Fusaro, A. F., Wang, M., and Waterhouse, P. M. 2005. RNA silencing platforms in plants. FEBS Lett. 579:5982-5987.

Whitfield, A. E., Ullman, D. E., and German, T. L. 2005. Tospovirus-thrips interactions. Annu. Rev. Phytopathol. 43:459-489.

Yu, S., and Pilot, G. 2014. Testing the efficiency of plant artificial microRNAs by transient expression in Nicotiana benthamiana reveals additional action at the translational level. Front. Plant Sci. 5:622.

\section{AUTHOR-RECOMMENDED INTERNET RESOURCES}

Clustal Omega multiple sequence alignment program: https://www.ebi.ac.uk/Tools/msa/clustalo

NCBI Nucleotide database: https://www.ncbi.nlm.nih.gov/nucleotide/ P-SAMS software: https://github.com/carringtonlab/p-sams

Solgenomics Solanum lycopersicum iTAGv2.3 index: ftp://ftp.solgenomics.net/tomato_genome/annotation/ITAG2.3_release

TargetFinder software: https:/github.com/carringtonlab/TargetFinder 